\title{
Effective Field Theory for the perturbations of a slowly rotating black hole
}

\author{
Lam Hui, ${ }^{a}$ Alessandro Podo, ${ }^{a}$ Luca Santoni ${ }^{a, b}$ and Enrico Trincherini ${ }^{c, d}$ \\ ${ }^{a}$ Department of Physics, Center for Theoretical Physics, Columbia University, \\ 538 West 120th Street, New York, NY 10027, U.S.A. \\ ${ }^{b}$ ICTP, International Centre for Theoretical Physics, \\ Strada Costiera 11, I-34151, Trieste, Italy \\ ${ }^{c}$ Scuola Normale Superiore, \\ Piazza dei Cavalieri 7, I-56126, Pisa, Italy \\ ${ }^{d}$ Istituto Nazionale di Fisica Nucleare (INFN) - Sezione di Pisa, \\ Polo Fibonacci, Largo B. Pontecorvo 3, I-56127 Pisa, Italy \\ E-mail: 1h399@columbia.edu, ap3964@columbia.edu, lsantoni@ictp.it, \\ enrico.trincherini@sns.it
}

ABSTRACT: We develop the effective theory for perturbations around black holes with scalar hair, in two directions. First, we show that the scalar-Gauss-Bonnet theory, often used as an example exhibiting scalar black hole hair, can be deformed by galileon operators leading to order unity changes to its predictions. The effective theory for perturbations thus provides an efficient framework for describing and constraining broad classes of scalartensor theories, of which the addition of galileon operators is an example. Second, we extend the effective theory to perturbations around an axisymmetric, slowly rotating black hole, at linear order in the black hole spin. We also discuss the inclusion of parity-breaking operators in the effective theory.

KEYwords: Black Holes, Effective Field Theories

ARXIV EPRINT: 2111.02072 


\section{Contents}

1 Introduction 2

2 Motivations for an effective theory 3

2.1 Black holes with galileon hair 3

2.1.1 Some preliminary scaling considerations 4

2.1.2 Background solution 5

$\begin{array}{ll}\text { 2.1.3 Linearized equations for the perturbations and quasi-normal modes } & 7\end{array}$

2.2 Isospectrality and eikonal limit in the EFT for the perturbations 8

3 Effective theory for perturbations of slowly rotating black holes $\quad 11$

3.1 General considerations and background metric 11

$\begin{array}{lll}3.2 & \text { Effective theory for the perturbations } & 13\end{array}$

$\begin{array}{lll}3.2 .1 & \text { Linear order } & 13\end{array}$

$\begin{array}{ll}3.2 .2 \text { Quadratic action } & 16\end{array}$

$\begin{array}{lll}4 & \text { Effective theory with parity-breaking operators } & 17\end{array}$

5 Conclusions 20

A Black holes with galileon hair: odd sector $\quad 22$

B Radial foliation and geometric decomposition $\quad 22$

B.1 Gauss-Codazzi equation 24

B.2 Geometric quantities evaluated on the background 24

C Infinitesimal variations $\quad \mathbf{2 4}$

$\begin{array}{ll}\text { C.1 Equivalent expressions for the } t \phi \text { tadpole } & 27\end{array}$

$\begin{array}{ll}\text { D Explicit example } & 27\end{array}$

E Vector and tensor spherical harmonics, and some useful identities $\quad 28$

$\begin{array}{lll}\text { E.1 Recurrence relations and other useful identities } & 29\end{array}$

$\begin{array}{lll}\text { F } & \text { Gauge choice } & 30\end{array}$

G Linearized equations of motion with even-odd mixing 32 


\section{Introduction}

Future gravitational wave experiments are expected to find not only a large number of black hole merger events, but also to measure their gravitational wave signals to high precision. One exciting prospect is the high quality data on black hole ring-down, sufficient to detect and measure multiple quasi-normal modes (QNMs) [1-3]. Each quasi-normal mode is characterized by the real and imaginary parts of its frequency, and thus one could put to the test the general relativistic prediction that black holes are completely characterized by their mass and spin (in addition to charge, which is generally expected to vanish). There are several different ways how such a test can be carried out. (1) The simplest one is a null test: are two numbers (mass and spin) sufficient to describe the observed quasi-normal spectrum? Beyond that, it is useful to test for the possible presence of deviations. (2) Concrete models help guide our thinking on the form of possible deviations and the associated model testing. The scalar-Gauss-Bonnet model (and its variations) is a popular example. It circumvents earlier no-scalar-hair theorems [4-7] and gives interesting predictions for the background geometry and its perturbations, see e.g. [8-13]. Another example is scalarChern-Simons model, see e.g. [14-26]. (3) A third approach is to parametrize the deviations from general relativistic expectations in a model-independent way. This can take the form of a phenomenological parametrization such as [27-32], or a parametrization at the level of the action governing the dynamics of black hole perturbations [33-37]. It is the last approach that is the subject of this paper. One main reason for our choice is that, as opposed to a phenomenological parametrization of possible deviations from general relativity, a parametrization at the level of an effective Lagrangian makes more transparent which types of deformations correspond to theories that respect physical principles, such as locality and diffeomorphism invariance, and makes it easier to connect UV theories with observations, by a systematic matching procedure.

An effective field theory for perturbations around a spherically symmetric spacetime with non-trivial scalar background was developed in [35, 38]. To orient ourselves, it is helpful to think of a perhaps more familiar example: the effective theory for perturbations around an inflating universe [39]. The goal of such a theory is not to explain where the (inflation) background comes from; rather, one takes the background as given and proceeds to write down an action that governs the dynamics of the perturbations, guided by the notions of symmetries and derivative expansion. To formulate an effective theory for black hole perturbations, we proceed in an analogous manner. It is assumed the black hole has a scalar background with a non-trivial radial profile, much like the inflaton having a non-trivial temporal profile. Exactly what (UV) physics gives rise to this scalar hair is immaterial for the construction of the effective theory. The important point is that one can choose a unitary gauge in which all the perturbations reside in the metric. In this gauge, we use the invariance under $t, \theta, \phi$-diffeomorphisms as the guiding principle for writing down the effective (IR) theory.

This strategy was adopted in [35] to construct an effective theory for perturbations around a spherically symmetric black hole with scalar hair. In this paper, we wish to address two follow-up questions: (1) What is the utility of an effective theory if known black 
hole hair solutions generally make use of a limited set of interactions (e.g. scalar-GaussBonnet, or scalar-Chern-Simons) - why not work directly with the UV theory instead of dealing with an effective IR theory? (2) Given that black holes in nature have angular momentum, can the effective theory be generalized to describe rotating black holes? We answer the first question by showing how the scalar-Gauss-Bonnet model can be deformed to yield a wide variety of predictions; an effective theory description is thus a useful way to parametrize the possibilities. We answer the second question by showing how the formalism of [35] can be adapted in a simple way to slowly rotating black holes. Black holes with a substantial spin is not as straightforward to work with - recall that even in general relativity, the only known way to write down separable equations of perturbations around a Kerr background is to use the Newman-Penrose variables, and that there is no known action formulation for the associated Teukolsky equation.

An outline of the paper is as follows. In section 2, we go over the motivations for the effective theory approach, using the scalar-Gauss-Bonnet model and its deformations as an example (focusing for simplicity on non-rotating black holes). In particular, we show how in the eikonal limit a judicious choice of the couplings in the effective theory allows one to recover isospectrality, which is usually broken in theories beyond general relativity. The point is not to motivate this rather finely-tuned choice of couplings, but to illustrate how a wide range of behavior is possible by deforming the scalar-Gauss-Bonnet model. We then develop the effective theory for perturbations around slowly rotating black holes in section 3. We discuss cases with parity-breaking operators in section 4 , and conclude in section 5. It is worth stressing that the resulting equations from the effective theory are often difficult to solve. The WKB approach provides an analytic and simple, even if approximate, way to deduce the quasi-normal spectrum [40-42]. In the even sector, however, one in general obtains a coupled set of equations governing the scalar and tensor modes and the application of the WKB approach is not as straightforward. In a follow-up paper [43], we will show how to analytically compute the (approximate) quasi-normal mode spectrum in such a coupled system, in a spirit similar to the WKB approximation.

Conventions. We work in mostly-plus signature for the metric, $(-,+,+,+)$, and in units where $c=\hbar=1$. We denote the reduced Planck mass with $M_{\mathrm{Pl}}=(8 \pi G)^{-1 / 2}$, where $G$ is the Newton's constant. We use Greek indices $\mu, \nu, \ldots$ to denote 4 -dimensional spacetime coordinates, while we use Latin indices $a, b, c, \ldots$ for temporal and angular coordinates $(t, \theta, \phi)$ only. Latin indices $i, j, \ldots$ label instead the angular coordinates $(\theta, \phi)$ on the $S^{2}$-sphere.

\section{Motivations for an effective theory}

\subsection{Black holes with galileon hair}

We discuss here an explicit example of hairy black hole where, in addition to the GaussBonnet coupling, the Lagrangian for the scalar contains another operator describing a particular higher derivative self-interaction of the field, which we show can induce orderone corrections to the solution of [9], where this operator is not included. We will use this example as a motivation for introducing an effective theory to study perturbations around 
black holes with hair: since the operator considered below is just one particular example of a much larger set of operators that can in principle affect the linearized dynamics for the perturbations and the observables, a model-independent approach is, in this sense, valuable.

For simplicity, the example discussed here is in the context of non-rotating black holes, though it can be straightforwardly generalized to the case of slowly rotating spacetimes.

\subsubsection{Some preliminary scaling considerations}

Let us consider the action

$$
S=\int \mathrm{d}^{4} x \sqrt{-g}\left[\frac{M_{\mathrm{Pl}}^{2}}{2} R-\frac{1}{2}(\partial \Phi)^{2}+g_{3} \frac{(\partial \Phi)^{2} \square \Phi}{\Lambda^{3}}+\alpha M_{\mathrm{Pl}} \Phi \mathcal{G}\right]
$$

In the limit $g_{3}=0$ one recovers the well studied case of $[9,10]$, where the scalar field $\Phi$ is linearly coupled to the Gauss-Bonnet term $\mathcal{G}$,

$$
\mathcal{G} \equiv R^{\mu \nu \rho \sigma} R_{\mu \nu \rho \sigma}-4 R^{\mu \nu} R_{\mu \nu}+R^{2} .
$$

In (2.1), we added as an additional operator the cubic galileon self-interaction $(\partial \Phi)^{2} \square \Phi[44]$, suppressed by a scale $\Lambda$ to be fixed later. A consistent low-energy Effective Field Theory (EFT) will of course contain infinitely many irrelevant operators whose coefficients scale accordingly to naive dimensional analysis. In this section, however, our goal is to show that at least some of those operators can be relevant in describing the background solution around black holes and the dynamics of perturbations in addition to the scalar GaussBonnet (sGB) coupling. For this purpose, it is enough to focus on a simple model, though there are in fact other operators that can be considered as well [45].

The choice of defining the coefficient of the linear coupling between $\Phi$ and the GaussBonnet invariant in terms of a coupling $\alpha$ with the dimension of length squared, as in (2.1), is common in the literature. It is particularly convenient because it immediately identifies the typical length below which corrections from the Gauss-Bonnet coupling become relevant. For a black hole, for example, it is easy to see that the space-time geometry close to the horizon is modified by corrections $\mathcal{O}\left(\alpha / r_{s}^{2}\right)$, where $r_{s}$ defines the position of the black hole horizon. Therefore, we find useful to introduce the dimensionless coupling

$$
\tilde{\alpha} \equiv \frac{\alpha}{r_{s}^{2}} \text {. }
$$

The definition adopted in (2.1), on the other hand, makes less transparent another relevant scale associated with that coupling. The sGB operator contains a series of irrelevant interactions between the scalar field and an arbitrary number of gravitons. The leading one is a cubic vertex, schematically of the form $\partial^{2} \Phi \partial h \partial h$. When the graviton field is canonically normalized, the interaction is a dimension 7 operator

$$
\frac{1}{\Lambda_{\alpha}^{3}} \partial^{2} \Phi \partial h_{c} \partial h_{c}
$$

where $\Lambda_{\alpha}$ is the energy at which the cubic interaction becomes strongly coupled. In terms of $\alpha$ one immediately gets

$$
\frac{1}{\Lambda_{\alpha}}=\left(\frac{\alpha}{M_{\mathrm{Pl}}}\right)^{1 / 3} .
$$


From now on, we will assume that the coupling $\alpha$ has the largest value allowed by present observations. The strongest bound on $\alpha$ comes from the absence of any signal of scalar wave dipolar emission, sourced by that coupling, in black hole merger data. A recent analysis finds $\alpha \lesssim(1.7 \mathrm{~km})^{2}$ [46] (see also refs. [12, 47]). In terms of the associated strong coupling scale the bound becomes

$$
\Lambda_{\alpha} \gtrsim 10^{12} \mathrm{~km}^{-1} .
$$

When these values are saturated, the coefficient parametrizing deviations from general relativity predictions for black holes in the LIGO-Virgo band is at most $\tilde{\alpha} \sim 0.1-0.01$.

We now turn to estimate the size of the effects induced by the additional galileon interaction. To do so, we have to fix the value of $\Lambda$ in (2.1), taking for simplicity $g_{3} \sim \mathcal{O}(1)$. We will make a natural assumption: all the leading interactions involving the scalar $\Phi$ become strong at the same scale. This implies that we have to set $\Lambda \sim \Lambda_{\alpha}$. From now on we will work under this assumption and discuss the implications of the cubic galileon coupling $g_{3}$ on the hairy black hole solution.

From the scalar's equations of motion, $\square \Phi\left(1+\frac{\square \Phi}{\Lambda^{3}}+\ldots\right) \sim \alpha M_{\mathrm{Pl}} \mathcal{G}$, one can see that the presence of the cubic interaction gives $\mathcal{O}(1)$ corrections to the background solution obtained for $g_{3}=0$ when

$$
\frac{\square \Phi}{\Lambda^{3}} \sim \mathcal{O}(1)
$$

Solving for the scalar field far from the horizon we can neglect the galileon interaction: the field is sourced by the curvature squared term evaluated on the Schwarzschild solution

$$
\square \Phi \sim \alpha M_{\mathrm{Pl}} \frac{r_{s}^{2}}{r^{6}} ;
$$

we can therefore estimate that at the horizon $r \sim r_{s}$

$$
\frac{\square \Phi}{\Lambda^{3}} \sim \frac{\alpha M_{\mathrm{Pl}}}{r_{s}^{4} \Lambda^{3}}
$$

that is $\mathcal{O}(1)$ for a black hole with $r_{s} \simeq 10 \mathrm{~km}$, using $\Lambda \simeq \Lambda_{\alpha} \simeq 10^{12} \mathrm{~km}^{-1}$ and $M_{\mathrm{Pl}} \simeq$ $10^{38} \mathrm{~km}^{-1}$. In conclusion we obtained that, with a natural choice for scale of the cubic interaction, close to the horizon there are large corrections to the solution. In the next section we will solve perturbatively in $\tilde{\alpha} \ll 1$ the equation of motion of the action (2.1). The perturbative expansion is schematically organized as follows,

$$
\begin{aligned}
\Phi & \sim M_{\mathrm{Pl}}\left(\tilde{\alpha}+\tilde{\alpha}^{3}+\ldots\right), \\
h & \sim \tilde{\alpha}^{2}+\tilde{\alpha}^{4}+\ldots,
\end{aligned}
$$

where $h$ denotes the metric fluctuations with respect to the Schwarzschild background.

\subsubsection{Background solution}

In this section, we will solve perturbatively the fields' equations of motion up to quadratic order in $\tilde{\alpha}$. The background metric can be parametrized as follows,

$$
\mathrm{d} s^{2}=-A(r) \mathrm{d} t^{2}+\frac{\mathrm{d} r^{2}}{B(r)}+r^{2}\left(\mathrm{~d} \theta^{2}+\sin ^{2} \theta \mathrm{d} \phi^{2}\right)
$$


where, in full generality, we set the coefficient of $\mathrm{d} \Omega_{S^{2}}^{2} \equiv \mathrm{d} \theta^{2}+\sin ^{2} \theta \mathrm{d} \phi^{2}$ to $r^{2}$, and where

$$
\begin{aligned}
& A(r)=1-\frac{r_{s}}{r}+\tilde{\alpha}^{2} \mathcal{A}(r)+\mathcal{O}\left(\tilde{\alpha}^{4}\right), \\
& B(r)=1-\frac{r_{s}}{r}+\tilde{\alpha}^{2} \mathcal{B}(r)+\mathcal{O}\left(\tilde{\alpha}^{4}\right) .
\end{aligned}
$$

The scalar field profile can be instead expanded as

$$
\bar{\Phi}(r)=M_{\mathrm{Pl}}\left(\tilde{\alpha} \varphi(r)+\mathcal{O}\left(\tilde{\alpha}^{3}\right)\right) .
$$

Plugging into the Einstein equations, one can find the equations for $\mathcal{A}(r), \mathcal{B}(r)$ and $\varphi(r)$. These do not usually admit a closed form solution if $g_{3} \neq 0$. However, integrating once the equation for $\varphi(r)$, one can find an expression for $\varphi^{\prime}(r)$ in closed form:

$$
\varphi^{\prime}(r)=\frac{r^{3}-\sqrt{r^{6}+16 g_{3}\left(4 r^{3} r_{s}^{3}+r^{2} r_{s}^{4}+r r_{s}^{5}-3 r_{s}^{6}\right)}}{2 g_{3} r r_{s}^{2}\left(4 r-3 r_{s}\right)},
$$

where we fixed the integration constant in such a way that $\varphi^{\prime}(r)$ is finite at the horizon (which is sufficient to guarantee $(\partial \varphi)^{2}$ is regular at the horizon) . Note that, in the limit $g_{3} \rightarrow 0$, one recovers the result of $[9]$,

$$
\left.\varphi^{\prime}(r)\right|_{g_{3} \rightarrow 0} \rightarrow-\frac{4 r_{s}\left(r^{2}+r r_{s}+r_{s}^{2}\right)}{r^{4}} .
$$

Regarding the equations for the metric components $\mathcal{A}(r)$ and $\mathcal{B}(r)$, it is convenient to look for solutions in the following form,

$$
\begin{aligned}
& \mathcal{A}(r)=\mathcal{B}(r)-\left(1-\frac{r_{s}}{r}\right) \int_{r}^{\infty} \mathrm{d} \rho \mathcal{F}_{A}(\rho), \\
& \mathcal{B}(r)=\frac{1}{r} \int_{r_{s}}^{r} \mathrm{~d} \rho \mathcal{F}_{B}(\rho),
\end{aligned}
$$

where $\mathcal{F}_{A}$ and $\mathcal{F}_{B}$ are lengthy expressions, which we do not write explicitly, that can be obtained straightforwardly by plugging (2.17) into the equations for $\mathcal{A}(r)$ and $\mathcal{B}(r)$. The integrals in (2.17) can be shown to be convergent for all $r \in\left(r_{s}, \infty\right){ }^{1}$ As a sanity check, it can also be shown that $\mathcal{A}$ and $\mathcal{B}$ agree with eqs. (3) and (4) of [21] in the limit $g_{3} \rightarrow 0$. In (2.17), the boundary conditions have been chosen in such a way that the position of the horizon is still at $r=r_{s}$ (i.e., $\mathcal{A}$ and $\mathcal{B}$ both vanish at $r_{s}$ ). Note that $\mathcal{A} \rightarrow \mathcal{B}$ as $r \rightarrow+\infty$, and they both scale as $\frac{1}{r}$. This implies a modification to the ADM mass with respect to general relativity and it is given by the formula

$$
2 G M=r_{s}-\tilde{\alpha}^{2} \int_{r_{s}}^{\infty} \mathrm{d} \rho \mathcal{F}_{B}(\rho),
$$

where the correction can be easily computed numerically for fixed $g_{3}$ and $r_{s}$.

Note that the scalar hair at large distances goes as (see (2.15))

$$
\varphi(r \rightarrow \infty) \rightarrow \frac{4 r_{s}}{r}+\ldots
$$

Corrections in $g_{3}$ only enter starting from $\frac{1}{r^{4}}$. This means, for instance, that the scalar charge $P \equiv \frac{4 \alpha}{r_{s}}$ is unchanged with respect to the case with $g_{3}=0$ of [9].

\footnotetext{
${ }^{1}$ One can show that both $\mathcal{F}_{A}(\rho)$ and $\mathcal{F}_{B}(\rho)$ are continuous functions of $\rho$. In addition, they both approach a constant at $r=r_{s}$, while at large distances $\mathcal{F}_{A}(\rho \rightarrow \infty) \sim \frac{1}{\rho^{3}}$ and $\mathcal{F}_{B}(\rho \rightarrow \infty) \sim \frac{1}{\rho^{2}}$.
} 


\subsubsection{Linearized equations for the perturbations and quasi-normal modes}

The additional operator in (2.1) not only modifies the background geometry, but it also induces order-one corrections to the linearized dynamics of the perturbations, as we show in this section. To this end, we will use the following conventions for the metric perturbations [35, 38],

$$
\delta g_{\mu \nu}=\delta g_{\mu \nu}^{\text {odd }}+\delta g_{\mu \nu}^{\text {even }}
$$

with

$$
\delta g_{\mu \nu}^{\text {odd }}=\left(\begin{array}{ccc}
0 & 0 & \varepsilon^{k}{ }_{j} \nabla_{k} \mathrm{~h}_{0} \\
0 & 0 & \varepsilon^{k}{ }_{j} \nabla_{k} \mathrm{~h}_{1} \\
\varepsilon^{k}{ }_{i} \nabla_{k} \mathrm{~h}_{0} & \varepsilon^{k}{ }_{i} \nabla_{k} \mathrm{~h}_{1} & \frac{1}{2}\left(\varepsilon_{i}{ }^{k} \nabla_{k} \nabla_{j}+\varepsilon_{j}{ }^{k} \nabla_{k} \nabla_{i}\right) \mathrm{h}_{2}
\end{array}\right)
$$

which parametrizes parity-odd perturbations and where $\varepsilon_{i j}$ is defined in (E.1), ${ }^{2}$ and

$$
\delta g_{\mu \nu}^{\text {even }}=\left(\begin{array}{ccc}
A H_{0} & H_{1} & \nabla_{j} \mathcal{H}_{0} \\
H_{1} & H_{2} / B & \nabla_{j} \mathcal{H}_{1} \\
\nabla_{i} \mathcal{H}_{0} & \nabla_{i} \mathcal{H}_{1} & C\left(\mathcal{K} \gamma_{i j}+\nabla_{i} \nabla_{j} G\right)
\end{array}\right)
$$

for perturbations of the even type. $\nabla_{i}$ denotes a covariant derivative on the 2-sphere $S^{2}$, and in standard coordinates with metric $\gamma_{i j} \equiv \operatorname{diag}\left(1, \sin ^{2} \theta\right)$ it follows that

$$
\begin{aligned}
\nabla_{\theta} \nabla_{\theta} & =\partial_{\theta}^{2}, \quad \nabla_{\phi} \nabla_{\phi}=\partial_{\phi}^{2}+\sin \theta \cos \theta \partial_{\theta}, \quad \nabla_{\theta} \nabla_{\phi}=\nabla_{\phi} \nabla_{\theta}=\partial_{\theta} \partial_{\phi}-\frac{\cos \theta}{\sin \theta} \partial_{\phi} \\
\nabla^{2} & =\partial_{\theta}^{2}+\frac{1}{\sin ^{2} \theta} \partial_{\phi}^{2}+\frac{\cos \theta}{\sin \theta} \partial_{\theta} .
\end{aligned}
$$

The scalar perturbations are instead parametrized as $\Phi=\bar{\Phi}+\delta \Phi$, where $\bar{\Phi}$ is given in (2.14). Given the spherical symmetry of the background, it is guaranteed that even and odd perturbations do not couple at the level of the linearized equations of motion. For this reason, we can study the two sectors separately. In addition, the spherical symmetry and the time-translational invariance of the background allow to decompose a generic field perturbation $\mathcal{X}$ as $\mathcal{X}(t, r, \theta, \phi)=\sum_{\ell m} \int \frac{\mathrm{d} \omega}{2 \pi} \mathrm{e}^{-i \omega t} \mathcal{X}_{\ell m}(\omega, r) Y_{\ell m}(\theta, \phi)$, where $Y_{\ell m}$ are the spherical harmonics and where $\mathcal{X}$ can be either $\delta \Phi$ or any of the components in $\delta g_{\mu \nu}$.

Let us start considering the odd sector, which is indeed the simplest one, since it contains only one propagating degree of freedom. Following the standard procedure, we shall first choose the Regge-Wheeler gauge, $\mathrm{h}_{2}=0$ [48]. ${ }^{3}$ Then, using (2.21) and the spherical harmonic decomposition, we can expand the action (2.1) up to quadratic order in the odd fields $\mathrm{h}_{0}(\omega, r)$ and $\mathrm{h}_{1}(\omega, r) .{ }^{4}$ It is then straightforward to compute the equations of motion for $h_{0}$ and $h_{1}$, and find the combination that is algebraic in $h_{0}$. Using this to solve

\footnotetext{
${ }^{2}$ Indices are raised and lowered with $\gamma_{i j} \equiv \operatorname{diag}\left(1, \sin ^{2} \theta\right)$.

${ }^{3}$ Fixing the Regge-Wheeler gauge, $\mathrm{h}_{2}=0$, at the level of the action (2.1) is consistent as one does not lose any constraints (see, e.g., [35]).

${ }^{4}$ For simplicity, we will hereafter suppress $\ell$ and $m$ subscripts in the spherical harmonic decomposition of the field perturbations, and also often altogether omit the arguments in the fields, i.e. $\mathcal{X}_{\ell m}(\omega, r)$ will simply be often denoted as $\mathcal{X}(\omega, r)$, or just as $\mathcal{X}$. That we are talking about fields decomposed in spherical harmonics and in frequency space should be clear from the context.
} 
for $\mathrm{h}_{0}$ in terms of $\mathrm{h}_{1}$ and $\mathrm{h}_{1}^{\prime}$, one arrives at a single equation for the propagating degree of freedom, which can be recast in the Schrödinger-like form

$$
\frac{\mathrm{d}^{2} Q}{\mathrm{~d} r_{\star}^{2}}+\left(\omega^{2}-V_{Q}\right) Q=0,
$$

where the tortoise coordinate $r_{\star}$ is defined by

$$
\frac{\mathrm{d} r_{\star}}{\mathrm{d} r}=\frac{1}{\sqrt{A B}},
$$

and where we introduced the field $Q$, which is related to $\mathrm{h}_{1}$ via

$$
\mathrm{h}_{1}(r) \equiv \mathrm{e}^{\mathcal{N}(r)} Q(r) \text {, }
$$

The explicit expressions for $\mathcal{N}(r)$ and the potential $V_{Q}(r)$ are given in appendix A. It is straightforward to check that, if $g_{3} \sim \mathcal{O}(1)$, the galileon coupling induces $\mathcal{O}(1)$ corrections to the potential $V_{Q}(r)$, with respect to [9], at distances $r \sim r_{s}$, and therefore modifies the spectrum of quasi-normal modes.

The even sector is more complicated, but can still be solved following, e.g., [35]. The result is a system of coupled equations of the form

$$
\frac{\mathrm{d}^{2} \vec{\psi}}{\mathrm{d} r_{\star}^{2}}+\left(\omega^{2}-\mathbb{V}_{\psi}\right) \vec{\psi}=0,
$$

where $\vec{\psi}$ is a 2-component vector, describing the two parity-even propagating degrees of freedom, and $\mathbb{V}_{\psi}$ is a $(2 \times 2)$-matrix. The relation between $\vec{\psi}$ and the perturbations $\delta \Phi$ and $\delta g_{\mu \nu}^{\text {even }}$ (see eq. (2.22)), the explicit expression for $\mathbb{V}_{\psi}$, as well as the detailed procedure to obtain (2.27), are included in the supplementary material of the present paper. In the notebook, we also compute explicitly the eikonal limit for the potential $\mathbb{V}_{\psi}$, which generalizes the result of [49].

\subsection{Isospectrality and eikonal limit in the EFT for the perturbations}

We have shown above how the presence of the cubic galileon operator modifies the properties of the hair generated by a sGB coupling. This example illustrates the possibility of including many other scalar operators in the theory. From this perspective, an effective field theory is particularly useful, if one is interested in studying the dynamics of the perturbations in a model-independent way. This is the approach that was first introduced in [35] and that we will pursue from now on. Before generalizing the effective theory of [35] to the case of slowly rotating black holes with scalar hair (see section 3 below), we consider here another application of [35] in the context of non-rotating black holes.

It is well known that the degeneracy between the even and odd QNM spectra of general relativity is broken in the presence of a scalar coupling to the Gauss-Bonnet operator. In the small-coupling limit, for the model of [9], this has been shown explicitly for low $\ell$ 's in [10] and in the eikonal limit in [49]. In this section, in the spirit of studying how additional operators in the EFT can affect the observables, we will show that the introduction of 
other operators can have phenomenological implications, altering the spectrum of the quasinormal modes. In particular we will show explicitly that suitable choices of the effective couplings allow to recover isospectrality in the eikonal limit. ${ }^{5}$ In this limit the equations simplify and the conditions under which isospectrality is recovered can be stated easily. The main point in this section is not to motivate the particular choice of couplings giving rise to isospectrality, but rather to illustrate, using the language of EFT, how a wide range of predictions is possible (such as whether isospectrality is satisfied or not).

Let us take the EFT of $[35,38]$ and let us focus on the following subset of operators,

$$
S=\int \mathrm{d}^{4} x \sqrt{-g}\left[\frac{M_{\mathrm{Pl}}^{2}}{2} R+\xi(r) \mathcal{G}-\Lambda(r)-f(r) g^{r r}+M_{2}^{4}(r)\left(\delta g^{r r}\right)^{2}+M_{13}^{2}(r) \delta g^{r r} \delta \hat{R}\right],
$$

where $\xi(r)$ encodes the scalar coupling to the Gauss-Bonnet invariant $\mathcal{G}$, while $\left(\delta g^{r r}\right)^{2}$ and $\delta g^{r r} \delta \hat{R}$ ( $\hat{R}$ is the intrinsic curvature associated with constant- $r$ hypersurfaces [35]) are additional quadratic operators in the fields that can be generated in theories with derivative self-interactions for the scalar. Recall that, as opposed to $M_{2}^{4}(r)$ and $M_{13}^{2}(r)$, which are in principle arbitrary, the coefficients $\Lambda(r)$ and $f(r)$ are fixed by the background equations of motion [35]. Since the procedure to obtain the field equations has already been extensively discussed in $[35,38]$ for non-rotating spacetimes, we will not repeat the derivation here, but we will simply highlight the main steps.

Instead of (2.12), we find it more convenient to parametrize the background metric as follows,

$$
\mathrm{d} s^{2}=-A(r) \mathrm{d} t^{2}+\frac{\mathrm{d} r^{2}}{A(r)}+C(r)\left(\mathrm{d} \theta^{2}+\sin ^{2} \theta \mathrm{d} \phi^{2}\right)
$$

where we set $A=B$ and kept the coefficient $C$ of the line element on the 2 -sphere arbitrary. The relation between the tadpole coefficients $f$ and $\Lambda$ and the background metric can be found by solving the background equations of motion [35, 38]. The equation for the odd mode can then be derived in complete analogy with section 2.1.3: we can choose the Regge-Wheeler gauge, $\mathrm{h}_{2}=0$, expand the action (2.28) up to quadratic order in the metric perturbations (2.21) and integrate out $\mathrm{h}_{0}$ at the level of the linearized equations of motion. The final result is an equation of the form,

$$
\frac{\mathrm{d}^{2} Q}{\mathrm{~d} r_{\star}^{2}}+\left(c_{r}^{-2} \omega^{2}-V_{Q}\right) Q=0
$$

where now $\frac{\mathrm{d} r_{\star}}{\mathrm{d} r}=A^{-1}$ and $c_{r}$ plays the role of an effective speed of propagation (which can in general be non-unitary) in the radial direction. In the eikonal limit, $\ell \rightarrow \infty$, the function $c_{r}^{-2}$ and the potential $V_{Q}$ are

$$
c_{r}^{-2}=\frac{M_{\mathrm{Pl}}^{2}-4 A^{\prime} \xi^{\prime}-8 A \xi^{\prime \prime}}{M_{\mathrm{Pl}}^{2}-4 A^{\prime} \xi^{\prime}}, \quad V_{Q}=\ell(\ell+1) \frac{A\left(M_{\mathrm{Pl}}^{2}-4 A^{\prime} \xi^{\prime}-8 A \xi^{\prime \prime}\right)}{M_{\mathrm{Pl}}^{2} C-4 A C^{\prime} \xi^{\prime}}+\mathcal{O}\left(\ell^{0}\right),
$$

\footnotetext{
${ }^{5}$ By 'isospectrality' we generically mean here that there is a degeneracy between even and odd sector at the level of the linearized equations of motion for the perturbations. Establishing whether or not there is an actual degeneracy at the level of the observed frequencies requires an additional piece of information, that we will not discuss in the present work, which is specifying how the scalar couples to the matter.
} 
where in $V_{Q}$ we neglected subleading terms in $1 / \ell$. We can further assume that deviations from Schwarzschild are small, i.e., $A \equiv 1-\frac{r_{s}}{r}+\epsilon \mathcal{A}$ and $C \equiv r^{2}+\epsilon \mathcal{C}$, where $\epsilon$ is some small coupling, and $\xi, M_{2}^{4}, M_{13}^{2} \sim \mathcal{O}(\epsilon)$. Under this assumption, eqs. (2.31) become

$$
\begin{aligned}
c_{r}^{-2} & =1-\left(1-\frac{r_{s}}{r}\right) \frac{8 \xi^{\prime \prime}}{M_{\mathrm{Pl}}^{2}}, \\
V_{Q} & =\frac{\ell(\ell+1)}{r^{2}}\left(1-\frac{r_{s}}{r}\right)\left[1+\frac{\epsilon \mathcal{A}}{1-\frac{r_{s}}{r}}-\frac{M_{\mathrm{Pl}}^{2} \mathcal{C}-4\left(2 r-3 r_{s}\right) \xi^{\prime}-8 r\left(r_{s}-r\right) \xi^{\prime \prime}}{r^{2} M_{\mathrm{Pl}}^{2}}\right] .
\end{aligned}
$$

Following [35, 38], one can derive the equations governing the dynamics of the even modes. After the non-dynamical fields are integrated out on-shell and after a suitable field redefinition that allows to remove first derivative terms in $r_{\star}$, the final system of equations can be cast in the following form:

$$
\frac{\mathrm{d}^{2} \vec{\psi}}{\mathrm{d} r_{\star}^{2}}+\mathbb{W}_{\psi} \cdot \vec{\psi}=0,
$$

where $\mathbb{W}_{\psi}$ is a $(2 \times 2)$-matrix that depends on $r$, as well as on the frequency and the quantum number $\ell$. Note that the two equations will remain in general coupled even in the eikonal limit, $\ell \rightarrow \infty$, as opposed to what happens in other situations, e.g., in the case of massive and partially massless higher-spin fields in a Schwarzschild (de Sitter) spacetime [50]. However, it is possible to choose the couplings $\xi, M_{2}^{4}$ and $M_{13}^{2}$ in such a way that the two equations (2.34) decouple. In the particular limit under consideration, this happens, for all $r$, if the following conditions are satisfied, ${ }^{6}$

$$
\begin{aligned}
M_{2}^{4} & =-\frac{12 r_{s}\left(\left(-2 r^{2}+4 r r_{s}-3 r_{s}^{2}\right) \xi^{\prime}+r\left(2 r^{2}-5 r r_{s}+3 r_{s}^{2}\right) \xi^{\prime \prime}\right)}{r^{3}\left(2 r-3 r_{s}\right)^{2}\left(r-r_{s}\right)}, \\
M_{13}^{2} & =-\frac{12 r_{s} \xi^{\prime}}{2 r^{2}-3 r r_{s}} .
\end{aligned}
$$

When eqs. (2.35) hold, one can show not only that one of the off-diagonal entries of $\mathbb{W}_{\psi}$ becomes zero, but also that the corresponding equation becomes identical to the odd equation (2.30). In particular, the coefficients of the $\omega^{2}$ terms and the potentials equal (2.32) and (2.33), respectively. ${ }^{7}$ In this sense, one recovers 'isospectrality' between the odd spectrum and one of the two even set of frequencies. ${ }^{8}$

Given the action (2.28) with the coefficients (2.35), and given some educated guess on the form of the scalar profile $\bar{\Phi}(r)$, one can in principle, by reverse engineering, find explicit examples of covariant Lagrangians that reduce to $(2.28)$ in unitary gauge. ${ }^{9} \mathrm{Al}-$ though possible in general, this procedure does not guarantee that the resulting effective

\footnotetext{
${ }^{6}$ We stress that we are first taking the large- $\ell$ limit in (2.34) and then expanding the result at linear order in $\epsilon$.

${ }^{7}$ The fact that the equations become identical is a result of the eikonal limit (the same happens for instance with the Regge-Wheeler and Zerilli equations in general relativity). Note that this is a sufficient condition for isospectrality, but it is not necessary. Different potentials can in fact lead to identical spectra, provided that they are related by a duality symmetry that preserves the boundary conditions [50-53].

${ }^{8}$ We stress, though, that the corresponding even mode is still in general a mixture of scalar and metric fluctuations (see also the comment in footnote 5). For analogous considerations in the case of scalar-tensor theories on exactly Schwarzschild background, see, e.g., [34].

${ }^{9}$ See [54] for a discussion, although in a different context, on how to obtain a reverse-engineered covariant model.
} 
scalar-tensor theory passes extra sanity checks, such us having a well-defined expansion around a Minkowski spacetime, or a consistent embedding in a unitary, causal and local UV theory [55]. Whether there exist explicit examples that pass all these tests is left for future work.

\section{Effective theory for perturbations of slowly rotating black holes}

In this section, we generalize the results of $[35,38]$ and construct an effective theory for perturbations around slowly rotating black holes with scalar hair. We assume here that parity is not broken and the scalar field is invariant under parity transformation - we will discuss the case of parity-breaking theories in section 4. Even though what we will mostly have in mind (and refer to) below are asymptotically flat, slowly rotating black holes, we stress that our construction applies more generally to any stationary, axisymmetric, slowly rotating spacetime, whether or not it is asymptotically flat or has a horizon, including more exotic backgrounds (see [38] for an application of the EFT to wormholes in the non-rotating limit).

\subsection{General considerations and background metric}

In general relativity, a prominent example of stationary and axisymmetric spacetime is given by the Kerr metric, whose line element in Boyer-Lindquist coordinates is (see, e.g., $[56])$

$$
\begin{aligned}
\mathrm{d} s^{2}= & -\left(1-\frac{r_{s} r}{\Sigma}\right) \mathrm{d} t^{2}-\frac{2 a r_{s} r}{\Sigma} \sin ^{2} \theta \mathrm{d} t \mathrm{~d} \phi+\frac{\Sigma}{\Delta} \mathrm{d} r^{2}+\Sigma \mathrm{d} \theta^{2} \\
& +\left(r^{2}+a^{2}+\frac{a^{2} r_{s} r}{\Sigma} \sin ^{2} \theta\right) \sin ^{2} \theta \mathrm{d} \phi^{2}
\end{aligned}
$$

where $^{10}$

$$
\Sigma \equiv r^{2}+a^{2} \cos ^{2} \theta, \quad \Delta \equiv r^{2}-r_{s} r+a^{2},
$$

and where $a$ is related to the angular momentum $J$ of the rotating black hole by

$$
J=M a .
$$

At linear order in the spin parameter $a$ the metric reduces to:

$$
\mathrm{d} s^{2}=-\left(1-\frac{r_{s}}{r}\right) \mathrm{d} t^{2}+\frac{\mathrm{d} r^{2}}{1-\frac{r_{s}}{r}}+r^{2}\left(\mathrm{~d} \theta^{2}+\sin ^{2} \theta \mathrm{d} \phi^{2}\right)-a \frac{2 r_{s}}{r} \sin ^{2} \theta \mathrm{d} t \mathrm{~d} \phi .
$$

In our discussion below, we wish to retain the interpretation of $a$ as the parameter related to the spin of the black hole by an equation similar to (3.3). This leads us to consider metrics that, as (3.4), are invariant under the transformations $\{t \rightarrow-t, a \rightarrow-a\}$ and $\{t \rightarrow$ $-t, \phi \rightarrow-\phi\}$. These symmetries, together with the assumption of a smooth $a \rightarrow 0$ limit, imply that the only term linear in $a$ is in the $(t, \phi)$ component of the metric. Motivated

\footnotetext{
${ }^{10}$ The quantity $r_{s}$ is related to the locations of the inner $\left(r_{-}\right)$and outer $\left(r_{+}\right)$horizons by $r_{ \pm}=\frac{r_{s}}{2} \pm$ $\sqrt{\frac{r_{s}^{2}}{4}-a^{2}}$.
} 
by this observation and the form of the components in (3.4), we will focus below on the following class of stationary, axisymmetric background metrics:

$$
\mathrm{d} s^{2}=-A(r) \mathrm{d} t^{2}+\frac{1}{B(r)} \mathrm{d} r^{2}+C(r)\left(\mathrm{d} \theta^{2}+\sin ^{2} \theta \mathrm{d} \phi^{2}\right)-2 a D(r) \sin ^{2} \theta \mathrm{d} t \mathrm{~d} \phi,
$$

where $A(r), B(r), C(r)$ and $D(r)$ are arbitrary functions of $r .^{11}$

One might wonder how general the ansatz (3.5) is. As shown in refs. [57, 58], eq. (3.5) represents, at linear order in $a$, the most general ansatz for a stationary, axisymmetric and asymptotically flat black hole solution with separable geodesic equations, that preserves the Kerr symmetries and reduces to a spherically symmetric background in the limit $a \rightarrow 0$. Note that an overall $\theta$-dependent conformal factor could in principle be included in (3.5) while still preserving the Kerr symmetries [58]. In that case, in the limit $a \rightarrow 0$, one would recover a metric that is conformally related to the Schwarzschild solution. In what follows, we will for simplicity disregard this possibility, although such a $\theta$-dependent conformal factor could be straightforwardly accounted for in the construction of the EFT. ${ }^{12}$ Similarly, we will not consider here the possibility of more general rotating metrics with arbitrary $\theta$-dependence in the components. As an additional motivation for our ansatz (3.5), we stress that (3.5) is a good solution in many explicit examples of slowly rotating hairy black holes (see for instance refs. [21, 59] and appendix D below).

Given the ansatz (3.5), one still needs to specify the form of the scalar profile that sources it. This will be relevant when we construct the EFT for the perturbations in the unitary gauge. Note that, although the scalar's energy-momentum tensor is expected to have the same symmetries of the background metric, this need not be the case, in principle, for the scalar $\bar{\Phi}$ itself. A simple example of this is given by shift-symmetric theories where the solution for $\bar{\Phi}$ contains a linear term in time, $\bar{\Phi} \equiv t+\varphi(r)$ (see, e.g., [6063]). Despite the explicit time dependence in the field, the time-translational invariance of the system follows from the shift invariance of the scalar action. ${ }^{13}$ In this section, we work under the simplifying assumption that the scalar background for $\Phi$ inherits the same symmetries of the metric (3.5). As a consequence, $\bar{\Phi}$ cannot depend explicitly on $t$, nor on $\phi$. In addition, invariance under $\{a \rightarrow-a, \phi \rightarrow-\phi\}$ forbids linear terms in $a$ in $\bar{\Phi}$. Moreover, we will assume that $\Phi$ is a scalar under parity, i.e. that it is invariant under $\{\theta \rightarrow \pi-\theta, \phi \rightarrow \phi+\pi\}$, so that parity is broken neither explicitly in the action nor spontaneously by the background. This tells us that $\bar{\Phi}$ cannot depend on $\theta$ (in a way that solves non-trivially the background Einstein equations). As a result, $\bar{\Phi}$ must be a function of $r$ only $(\bar{\Phi} \equiv \bar{\Phi}(r))$ and with no dependence on $a$ at linear order [9]. We will partially relax these assumptions in section 4 , where we will allow the breaking of parity (in such a way that $\bar{\Phi}$ can depend on $\theta$ ) and the scalar $\bar{\Phi}$ to not be invariant under $\{a \rightarrow-a, \phi \rightarrow-\phi\} .{ }^{14}$

\footnotetext{
${ }^{11}$ In full generality, we are allowed, by the possibility of redefining the radial coordinate $r$, to fix one of the four functions $A(r), B(r), C(r), D(r)$.

${ }^{12} \mathrm{Such}$ a $\theta$-dependent conformal factor is strongly constrained by weak-field solar system observations, so that we can safely neglect it $[57,58]$.

${ }^{13}$ There is in fact a diagonal combination of time translations and constant shifts that is still linearly realized on $\Phi$.

${ }^{14}$ It would also be interesting to study more general cases and generalize the EFT to perturbations around
} 


\subsection{Effective theory for the perturbations}

To construct the EFT for the perturbations, we will work in the unitary gauge, defined by $\delta \Phi \equiv 0$. Since the scalar background $\bar{\Phi}$ depends on $r$, the unitary gauge breaks radial diffeomorphisms. This suggests the introduction of a radial foliation with hypersurfaces satisfying $\bar{\Phi}=$ constant, provided that the scalar profile is non-trivial, i.e., $\nabla_{\mu} \bar{\Phi} \neq 0$, and that the original map $\bar{\Phi}: r \rightarrow \bar{\Phi}(r)$ is an injective map on the whole domain in which the radial coordinate $r$ is defined. The continuity of $\bar{\Phi}(r)$, together with the previous conditions, implies that $\bar{\Phi}(r)$ has to be a strictly monotonic function in order to have a well-defined foliation.

After choosing the unitary gauge and fixing the radial foliation, the effective action will be the most general action invariant, up to boundary terms, under the residual temporal and angular diffeomorphisms. In analogy with [35, 38], the geometric objects that constitute the basic building blocks are $g_{\mu \nu}, R_{\mu \nu \rho \sigma}, \nabla_{\mu}, g^{r r}$ and the extrinsic curvature $K_{\mu \nu}$, with coefficients that can be arbitrary functions of $r$. For more details about the notation and the geometric decomposition, see [35] and appendices $\mathrm{B}$ and $\mathrm{C}$ below.

\subsubsection{Linear order}

In this section, we focus on the terms of the EFT that are linear in the field perturbations and find a set of independent operators that form a basis.

At linear order in perturbations, the most general action is

$$
S_{\text {tadpoles }}=\int \mathrm{d}^{4} x \sqrt{-g}\left[-\Lambda(r)-f(r) \delta g^{r r}+k^{\mu \nu}(r) \delta K_{\mu \nu}+\xi^{\mu \nu \alpha \beta}(r) \delta R_{\mu \nu \alpha \beta}\right],
$$

with $\Lambda(r), f(r), k^{\mu \nu}(r), \xi^{\mu \nu \alpha \beta}(r)$ arbitrary functions of the background metric and its derivatives.

Let us start considering the tadpole $k^{\mu \nu}(r) K_{\mu \nu}$. The orthogonality condition (B.7) implies that the only non-zero part is $k^{a b}(r) K_{a b}$. The function $k^{a b}$ is secretly a function of the background metric and thus inherits its structure. Therefore we can write:

$$
k^{a b}(r) \delta K_{a b}=k^{t t}(r) \delta K_{t t}+k^{\theta \theta}(r)\left(\delta K_{\theta \theta}+\frac{\delta K_{\phi \phi}}{\sin ^{2} \theta}\right)+a k^{t \phi}(r) \frac{\delta K_{t \phi}}{\sin ^{2} \theta} .
$$

Using the fact that the trace of $K=\nabla_{\mu} n^{\mu}$ is a total derivative and can be recast, up to a total derivative, as the $\Lambda(r)$ and $f(r) g^{r r}$ terms, we can get rid of one of the components in (3.7). For instance we can add a term $-k^{\theta \theta}(r) C(r) K$ and get rid of the second term in (3.7). We are left with two terms, which can be conveniently expressed as

$$
k^{a b}(r) K_{a b} \equiv \alpha(r) \bar{K}_{\mu \nu} \delta K^{\mu \nu}+a \sin ^{2} \theta \beta(r) \delta K^{t \phi} .
$$

The first term in (3.8) was already present in the EFT for non-rotating black holes [35]. The second term, proportional to the spin parameter $a$, is instead new and accounts for the fact that the spacetime is 'less symmetric', compared to the non-rotating case, which translates into a larger set of independent operators in the EFT.

Let us now consider the tadpole $\xi^{\mu \nu \alpha \beta}(r) R_{\mu \nu \alpha \beta}$. As before, $\xi^{\mu \nu \alpha \beta}(r)$ is secretly a function of the background metric and its derivatives, which dictate therefore its tensor solutions such that $\bar{\Phi} \equiv t+\varphi(r)$. This is left for future work. 
structure; moreover it has the same symmetry properties as $R_{\mu \nu \alpha \beta}$. This fact, together with the structure of the background metric, implies that out of the 20 components of the Riemann tensor, only 6 could give rise to new tadpoles:

$$
R_{r t r t}, \quad\left(R_{\theta r \theta r}+R_{\phi r \phi r}\right), \quad\left(R_{\theta t \theta t}+R_{\phi t \phi t}\right), \quad R_{\theta \phi \theta \phi}, \quad R_{r t r \phi}, \quad R_{\theta t \theta \phi} .
$$

We will show in fact that there is only one independent operator, corresponding to the Ricci scalar $R$. The first four terms in (3.9) are the same as those appearing in [35] for non-rotating spacetimes. Three of them can be re-expressed in terms of $R$, the other tadpoles or boundary terms - see appendix A of [35]. The last two tadpoles $R_{r t r \phi}, R_{\theta t \theta \phi}$ can instead be dealt with as follows. Since they both have $t \phi$ indices and are symmetric in their exchange, they will necessarily be contracted with $\bar{g}_{t \phi}$ or its derivatives, which is already order- $a$. Since we are interested in the EFT at linear order in $a$, we are thus allowed to think of $R_{r t r \phi}$ and $R_{\theta t \theta \phi}$ as being computed at the leading order $a=0$. Then, from eq. (C.5) we can read off the variation of the Riemann tensor at the order $a=0$, that is

$$
\begin{aligned}
& \delta R_{\theta t \theta \phi}=\bar{g}_{\theta \theta}\left(\nabla_{\theta} \delta \Gamma_{t \phi}^{\theta}-\nabla_{\phi} \delta \Gamma_{t \theta}^{\theta}\right), \\
& \delta R_{r t r \phi}=\bar{g}_{r r}\left(\nabla_{r} \delta \Gamma_{t \phi}^{r}-\nabla_{\phi} \delta \Gamma_{t r}^{r}\right) .
\end{aligned}
$$

The terms with covariant derivatives in $\theta$ and $\phi$ can be eliminated, up to boundary terms, by integrations by parts, while the term with an $r$ derivative can be also expressed as a $t \phi$ tadpole by using equation (C.21).

As a result, the most general tadpole action boils down to

$$
S_{\text {tadpoles }}=\int \mathrm{d}^{4} x \sqrt{-g}\left[\frac{1}{2} M_{1}^{2}(r) R-\Lambda(r)-f(r) g^{r r}-\alpha(r) \bar{K}^{\mu \nu} K_{\mu \nu}-a \sin ^{2} \theta \beta(r) g^{t \phi}\right],
$$

where, for convenience, we used $g^{t \phi}$ instead of $K^{t \phi} .15$

The coefficients in (3.11) are not all arbitrary. We report the form of the background equations in the case where $M_{1} \equiv M_{\mathrm{Pl}}$ is taken to be constant. The $t t$ and $r r$-Einstein equations fix $f(r)$ and $\Lambda(r):{ }^{16}$

$$
\begin{aligned}
f(r)= & \left(\frac{A^{\prime} C^{\prime}}{4 A C}-\frac{B^{\prime} C^{\prime}}{4 B C}+\frac{C^{2}}{4 C^{2}}-\frac{C^{\prime \prime}}{2 C}\right) M_{\mathrm{Pl}}^{2} \\
& +\left(\frac{A^{\prime} B^{\prime}}{A B}+\frac{2 A^{\prime} C^{\prime}}{A C}-\frac{4 A^{\prime 2}}{A^{2}}+\frac{2 A^{\prime \prime}}{A}-\frac{2 C^{\prime 2}}{C^{2}}\right) \frac{\alpha}{8}+\frac{A^{\prime}}{4 A} \alpha^{\prime}, \\
\Lambda(r)= & \left(-\frac{B A^{\prime} C^{\prime}}{4 A C}-\frac{B^{\prime} C^{\prime}}{4 C}-\frac{B C^{\prime \prime}}{2 C}+\frac{1}{C}\right) M_{\mathrm{Pl}}^{2} \\
& +B\left(\frac{A^{\prime} B^{\prime}}{A B}+\frac{2 A^{\prime} C^{\prime}}{A C}-\frac{4 A^{\prime 2}}{A^{2}}+\frac{2 A^{\prime \prime}}{A}-\frac{2 C^{\prime 2}}{C^{2}}\right) \frac{\alpha}{8}+\frac{B A^{\prime}}{4 A} \alpha^{\prime} .
\end{aligned}
$$

\footnotetext{
${ }^{15}$ The two operators are equivalent up to integrations by parts - see appendix C.1.

${ }^{16}$ Note that eqs. (3.12) and (3.14) here match eqs. (2.11)-(2.13) of [35], which are valid in the non-rotating limit $a=0$.
} 
In addition, the $t \phi$-component of the background Einstein equations can be used to derive an expression for $\beta(r)$ :

$$
\begin{aligned}
\beta(r)= & B D\left(-\frac{A^{\prime} B^{\prime}}{4 A B}-\frac{A^{\prime} D^{\prime}}{4 A D}+\frac{A^{\prime 2}}{4 A^{2}}-\frac{A^{\prime \prime}}{2 A}+\frac{B^{\prime} D^{\prime}}{4 B D}-\frac{1}{B C}+\frac{D^{\prime \prime}}{2 D}\right) M_{\mathrm{Pl}}^{2} \\
& +B D\left(-\frac{A^{\prime} B^{\prime}}{4 A B}+\frac{A^{\prime} C^{\prime}}{2 A C}-\frac{3 A^{\prime} D^{\prime}}{4 A D}+\frac{3 A^{\prime 2}}{4 A^{2}}-\frac{A^{\prime \prime}}{2 A}+\frac{B^{\prime} D^{\prime}}{4 B D}-\frac{C^{\prime} D^{\prime}}{2 C D}+\frac{D^{\prime \prime}}{2 D}\right) \alpha \\
& +B D\left(\frac{D^{\prime}}{D}-\frac{A^{\prime}}{A}\right) \frac{\alpha^{\prime}}{2},
\end{aligned}
$$

while the $\theta \theta$ (or $\phi \phi$ ) equation yields the following differential equation for $\alpha(r)$ :

$$
\begin{aligned}
\left(\frac{A^{\prime}}{A}-\frac{C^{\prime}}{C}\right) & \alpha^{\prime}+\left(\frac{A^{\prime} B^{\prime}}{2 A B}+\frac{A^{\prime} C^{\prime}}{2 A C}-\frac{3 A^{\prime 2}}{2 A^{2}}+\frac{A^{\prime \prime}}{A}-\frac{B^{\prime} C^{\prime}}{2 B C}+\frac{C^{2}}{C^{2}}-\frac{C^{\prime \prime}}{C}\right) \alpha \\
& +\left(\frac{A^{\prime} B^{\prime}}{2 A B}+\frac{A^{\prime} C^{\prime}}{2 A C}-\frac{A^{\prime 2}}{2 A^{2}}+\frac{A^{\prime \prime}}{A}-\frac{B^{\prime} C^{\prime}}{2 B C}+\frac{2}{B C}-\frac{C^{\prime \prime}}{C}\right) M_{\mathrm{Pl}}^{2}=0 .
\end{aligned}
$$

In the subset of theories in which $\alpha(r)=\beta(r) \equiv 0$ - corresponding to theories with no higher derivative operators - the background equations simplify and reduce to those of ref. [35], plus a self-consistency differential equation that relates the function $D(r)$ to the other background functions. Choosing coordinates in which $A(r)=B(r)$, this equation takes a particularly simple form:

$$
D^{\prime \prime}(r) C(r)-D(r) C^{\prime \prime}(r)=0
$$

Using this equation it is easy to generalise known examples of spherically symmetric hairy black holes to slowly rotating ones. An explicit realization within this type of theories is given in appendix D.

Since the most studied example of scalar hair in the literature is the one sourced by a coupling to the Gauss-Bonnet invariant, it might be sometimes convenient to isolate the Gauss-Bonnet operator, as we did already in eqs. (2.1) and (2.28):

$$
S_{\text {tad. }}=\int \mathrm{d}^{4} x \sqrt{-g}\left[\frac{1}{2} M_{1}^{2}(r) R+\xi(r) \mathcal{G}-\Lambda(r)-f(r) g^{r r}-\alpha(r) \bar{K}^{\mu \nu} K_{\mu \nu}-a \sin ^{2} \theta \beta(r) g^{t \phi}\right] .
$$

With this choice there is clearly a redundancy in the effective Lagrangian at linear order in perturbations, because not all the operators in (3.16) are independent, as we showed above. However, if the goal is to apply the EFT to study perturbations around black hole solutions with scalar hair, the choice (3.16) is particularly convenient. It allows, indeed, to more easily perform the matching with explicit models that involve a coupling to the Gauss-Bonnet invariant, which is one of the most studied examples in the literature evading the no-hair theorem. In fact, ref. [7] showed that in the context of scalar-tensor theories with no ghost degrees of freedom the no-hair theorem of Hui and Nicolis [4] can be evaded only in the presence of the operator $\Phi \mathcal{G}$, that is a linear coupling of the scalar to the 
Gauss-Bonnet invariant. In this sense, the EFT is capturing all possible corrections to the Einstein-Hilbert action and the sGB coupling. ${ }^{17}$

The general set of background equations in this parametrization and with all the tadpoles included is quite cumbersome. We therefore do not write them here, but make the derivation and result available in the supplementary material.

\subsubsection{Quadratic action}

In this section, we extend the action (3.16) to higher orders in the fields. In particular, we introduce quadratic operators, which we will use to study the linearized dynamics of the perturbations around black hole solutions with hair. We will omit the derivation, which follows the one in [35], of the complete set of operators that enter at quadratic order in the EFT. Instead, we will focus on a particular example and show how the linearized dynamics gets modified in the presence of the considered operator.

The linearized field equations on static spherically symmetric spacetimes are in general amenable to a separation of variables and a decomposition in spherical harmonics. For axisymmetric rotating backgrounds, it is in many cases also possible to decompose the fields in radial and angular components, provided that the spherical harmonics are suitably replaced by (spin-weighted) spheroidal harmonics. This is for instance the case of massless perturbations of generic spin $s$ on a Kerr background [64, 65]. However, at the linear order in the black hole spin parameter $a$, the situation is much simpler: the ansatz (2.20), with $\delta g_{\mu \nu}^{\text {odd }}$ and $\delta g_{\mu \nu}^{\text {even }}$ given in (2.21) and (2.22), remains a good parametrization of the metric perturbations; in addition, since the fields defining the components of $\delta g_{\mu \nu}$ in (2.21) and (2.22) transform as scalars, they can still be decomposed in spherical harmonics, in complete analogy with the non-rotating case $(a=0)$. The only main difference is that the linearized equations for $\delta g_{\mu \nu}^{\text {odd }}$ and $\delta g_{\mu \nu}^{\text {even }}$ will no longer be decoupled, with mixing terms between even and odd components appearing at linear order in $a$ (see, e.g., [66]). We refer the reader to appendices $\mathrm{E}$ and $\mathrm{F}$ for a detailed discussion of this decomposition and our choice of gauge. In the following, we derive the coupled equations for our EFT at the leading order in derivatives, showing that the result correctly reproduces the non-rotating case studied in $[35,38]$.

The simplest non-trivial example of quadratic operator is $\left(\delta g^{r r}\right)^{2}$. At the leading order in the derivative expansion, the EFT takes on the following simple form: ${ }^{18}$

$$
S=\int \mathrm{d}^{4} x \sqrt{-g}\left[\frac{1}{2} M_{\mathrm{Pl}}^{2} R-\Lambda(r)-f(r) g^{r r}+M_{2}^{4}(r)\left(\delta g^{r r}\right)^{2}\right] .
$$

To obtain the quadratic action for the propagating degrees of freedom (for generic $\ell$, one scalar and the two graviton polarizations), we shall proceed as follows. We first parametrize the fluctuations of the metric as in (2.20), (2.21) and (2.22); the residual gauge freedom allows us to get rid of 3 out of the 10 independent field variables; we plug (2.20) into (3.17)

\footnotetext{
${ }^{17}$ The fact of writing explicitly the Gauss-Bonnet operator in the effective action (3.16) should not be surprising. Note that we always do the same with the Einstein-Hilbert term. Given the special role played in the theory, it is just a matter of convenience to write it fully non-linearly, instead of expanding it in perturbations.

${ }^{18}$ An explicit model belonging to the class of theories described by (3.17) with $M_{2}^{4}=0$ is discussed in appendix D. Other examples captured by (3.17) with $M_{2}^{4}=0$ are also discussed in $[6,59]$.
} 
and expand up to quadratic order in the fields; we 'integrate out' the 4 non-dynamical fields and compute the 3 remaining equations for the propagating degrees of freedom. The derivation of the general set of coupled equations in the case of (3.17) is outlined in appendix G. Here, we emphasize that, as we discussed above and it is clear from appendix G, the linearized equations now mix even and odd modes. In particular, the equations of the even modes with fixed $\ell$ contain a coupling to the odd field with $\ell \pm 1$, and vice versa [67].

Since the mixing terms between odd and even modes are of order $\mathcal{O}(a)$, they generate corrections to the quasi-normal spectrum at quadratic order in $a$. Therefore, if one is interested in computing the QNMs at linear order in the spin parameter, these mixing terms can be neglected and the equations simplify considerably $[68,69]$. In this spirit, we show now the form of the odd equation if we neglect the coupling to the even modes. For simplicity let us set $B=A$ in (3.5) and let us fix the gauge where $\mathrm{h}_{2}=0$ in (2.21) (see appendix $\mathrm{F}$ for more details). As it is clear from (2.21), the coefficient $M_{2}^{4}$ will not enter the dynamics of the odd sector. Following the procedure outlined in appendix G, after straightforward manipulations, we arrive at the following equation for the axial mode:

$$
\frac{\mathrm{d}^{2} Q}{\mathrm{~d} r_{\star}^{2}}+\left(\omega^{2}-\frac{2 a m \omega D}{C}-V_{Q}\right) Q=0
$$

where $\frac{\mathrm{d} r_{\star}}{\mathrm{d} r}=A^{-1}=B^{-1}$ and where we defined

$$
\mathrm{h}_{1} \equiv \frac{\sqrt{C}}{A}\left(1-\frac{a m D}{\omega C}\right) Q .
$$

The potential $V_{Q}$ is given by

$$
\begin{aligned}
V_{Q}= & \frac{4\left(\ell^{2}+\ell-3\right) A C-2 A C A^{\prime} C^{\prime}-2 A C^{2} A^{\prime \prime}+3 A^{2} C^{2}}{4 C^{2}} \\
& +\frac{2 a m A\left(C A^{\prime}-3 A C^{\prime}\right)\left(C D^{\prime}-D C^{\prime}\right)}{\ell(\ell+1) \omega C^{3}}
\end{aligned}
$$

Note that, in the limit of a slowly rotating Kerr spacetime, i.e. $A=B=1-\frac{r_{s}}{r}, C=r^{2}$ and $D=\frac{r_{s}}{r}$, we recover precisely the equation of motion of the axial mode in general relativity (see, e.g., eq. (115) of [66]).

\section{Effective theory with parity-breaking operators}

In section 3 we showed how to construct the effective theory for the perturbations of slowly rotating hairy black holes under the assumption that parity is not broken. It is instructive to see how the previous conclusions change and what the EFT looks like for black hole solutions whose hair is sourced by a field profile that is not invariant under parity transformations. One notable example in this class of theories is given by dynamical ChernSimons gravity, where the (psuedo) scalar $\Phi$ couples linearly to the Pontryagin density ${ }^{*} R^{\mu \nu \rho \sigma} R_{\mu \nu \rho \sigma} \equiv \frac{1}{2} \varepsilon^{\mu \nu}{ }_{\lambda \tau} R^{\lambda \tau \rho \sigma} R_{\mu \nu \rho \sigma}[70,71]$. Both static and rotating black hole solutions in dynamical Chern-Simons gravity, as well as the analysis of the perturbations, have been widely discussed in the literature $[14-22,24-26]$. In this section, we extend the effective 
framework introduced in section 3 in such a way to capture, in a model-independent way, also modifications of gravity induced by couplings to parity-odd operators, like the ChernSimons one. The only assumption we will need is that of a well-defined radial foliation, that is: for every fixed value of the angular coordinate $\theta_{\star}$, the scalar background $\bar{\Phi}\left(r, \theta_{\star}\right)$ needs to be a strongly monotonic function of $r$ defined on the whole domain of definition of the radial coordinate $r$. The EFT will allow us to describe, in a single framework, many cases previously studied in the literature, as well as more general situations that have not been discussed before. As we will see in a moment, the purely dynamical Chern-Simons gravity with no additional operators is, however, an exception.

To construct the EFT for the perturbations, we assume again the parametrization (3.5) for the background metric. Even though its form is unchanged with respect to the case considered in section 3 , we now allow the background field $\bar{\Phi}$ to acquire some dependence on the angle $\theta$. Assuming that in the zero spin limit, $a=0$, one recovers the results of [35], the $\theta$-dependence will always be proportional to $a$. Thus, we can postulate the following generic profile for the background of $\Phi$,

$$
\bar{\Phi}(r, \theta)=\bar{\Phi}_{0}(r)+a \bar{\Phi}_{1}(r, \theta) .
$$

The form of $\Phi_{1}$ as function of $\theta$ depends on the model and is in general determined by the background equations of motion. In the case of dynamical Chern-Simons gravity (with no additional operators) the scalar background takes the simple form $\left.\bar{\Phi}(r, \theta)\right|_{\mathrm{dCS}}=$ $a f_{\mathrm{dCS}}(r) \cos \theta$. This implies $\left.\bar{\Phi}(r, \pi / 2)\right|_{\mathrm{dCS}} \equiv 0$, so that dynamical Chern-Simons gravity turns out to be a pathological example for our construction, since the scalar field cannot be used to define a radial foliation. In more general theories, however, one expects additional operators beyond the Chern-Simons one, and in general $\Phi_{0}(r) \neq 0 .{ }^{19}$ Explicit examples of slowly rotating black hole solutions in theories that include a coupling to the Pontryagin density plus additional operators are discussed e.g. in [21]. In these examples both the metric and the scalar background profiles are of the form of our ansatz (3.5) and (4.1).

Given the background profiles (3.5) and (4.1), we would like now to generalize the construction of the EFT presented in section 3. In addition to $g_{\mu \nu}, R_{\mu \nu \rho \sigma}, K_{\mu \nu}, \nabla_{\mu}$ and $g^{r r}$ that constitute the building blocks of the EFT in section 3, we are now allowed to contract spacetime indices also with the totally antisymmetric tensor $\varepsilon^{\mu \nu \rho \sigma}$. This gives more freedom and extend the number of independent operators in the EFT at each order in perturbation theory. The construction of the Lagrangian for the tadpoles closely follows the logic in section 3.2.1. One can similarly fix the unitary gauge by fixing the $r$-diffeomorphisms in such a way to remove the $\delta \Phi$ fluctuations altogether. The main difference in the construction of the foliation is that now the unit vector $n_{\mu}$, defined in (B.1), will have also a nonzero $\theta$-component at linear order in $a$, due to the non-trivial $\theta$-dependence in $\bar{\Phi}$. This implies, for instance, that the extrinsic curvature $K_{\mu \nu}$ can have now nonzero contravariant $r$-components at linear order in $a$ (see eqs. (B.6) and (B.7)). This modifies some of the

\footnotetext{
${ }^{19}$ When considering the EFT in the narrower context of hairy black holes in shift-symmetric scalar-tensor theories, a scalar profile with $\Phi_{0} \neq 0$ and $\Phi_{1} \neq 0$ requires the presence of a linear coupling of the scalar field to both the Gauss-Bonnet invariant and the Pontryagin density [7].
} 
steps in section 3.2.1, making the derivation of the tadpole Lagrangian slightly more complicated. Thus, instead of following the same logic, we provide here a simpler way to count the number of independent operators in the EFT at the linear order in perturbations. In the unitary gauge, the most general theory of a black hole with (pseudo-)scalar hair (4.1) can be now written in the form

$$
S=\int \mathrm{d}^{4} x \sqrt{-g} \mathcal{L}\left(g_{\mu \nu}, \varepsilon^{\mu \nu \lambda \rho}, R_{\mu \nu \alpha \beta}, g^{r r}, g^{r \theta}, K_{\mu \nu}, \nabla_{\mu} ; r, \theta\right),
$$

where the dependence on $g^{r \theta}$ and $\theta$ follows from the new $\theta$-dependent profile (4.1). When expanded in perturbations, up to integrations by parts, the Lagrangian at linear order can be in general recast in the form

$$
\mathcal{L}_{\text {tadpoles }} \sim F_{\mu \nu}\left[\partial_{r}, \partial_{\theta}, \bar{g}, \varepsilon ; r, \theta\right] \delta g^{\mu \nu},
$$

where the matrix $F_{\mu \nu}$ inherits its structure from the ingredients in (4.2) suitably contracted and computed on the background, and from the integrations by parts. In other words, it can be thought of as being some generic (symmetric) matrix resulting from certain contractions among the tensor $\varepsilon^{\mu \nu \lambda \rho}$, the spacetime derivatives $\partial_{\mu}$ projected on the $r$ and $\theta$-directions, and a certain number of background metric tensors $\bar{g}$. Thus, counting the number of independent tadpoles in (4.3) amounts in the end to finding the number of independent components in $F_{\mu \nu} \cdot{ }^{20}$ By definition, $\varepsilon^{\mu \nu \lambda \rho}$ is nonzero only when its indices are all different. In addition, since the background is independent of $t$ and $\phi$ by construction, no $t$ or $\phi$ derivatives can appear in $F$. Thus, given the form of $\bar{g}_{\mu \nu}$ in (3.5), it follows that the only metric fluctuations in (4.3) that allow to contract the Lorentz indices in a consistent way are

$$
\mathcal{L}_{\text {tadpoles }} \supseteq \delta g^{t t}, \delta g^{r r},\left(\delta g^{\theta \theta}+\sin ^{2} \theta \delta g^{\phi \phi}\right), \delta g^{t \phi}, \delta g^{r \theta}
$$

with generic coefficients that can depend on $r$ and $\theta$. As an illustrative example, let us assume that $F$ contains a single factor of $\varepsilon^{\operatorname{tr} \theta \phi}$. It is easy to envision how to obtain, for instance, $\delta g^{t \phi}$ by contracting the $r$ and $\theta$ indices in $\varepsilon^{\operatorname{tr} \theta \phi}$ with an $r$ and a $\theta$-derivative (recall that every index of each type must appear an even number of times). On the other hand, it is clearly not possible to generate $\delta g^{t r}$ in (4.4) at linear order in $a$, given the ansatz (3.5). As a result, the only tadpoles are the ones shown in (4.4). Using the fact that the trace of $g_{\mu \nu}$ is a constant, one can conveniently trade one of tadpoles in (4.4) for a generic function of $r$ and $\theta$ at the zero-th order in perturbations. The final result is thus, schematically,

$$
S_{\text {tadpoles }} \supseteq \int \mathrm{d}^{4} x \sqrt{-g}\left[\Lambda(r, \theta)+f_{1}(r, \theta) \delta g^{r r}+f_{2}(r, \theta) \delta g^{t t}+a f_{3}(r, \theta) \delta g^{t \phi}+a f_{4}(r, \theta) \delta g^{r \theta}\right],
$$

where we emphasized that $\delta g^{t \phi}$ and $\delta g^{r \theta}$ start linearly in $a$. In conclusion, the number of independent operators, up to linear order in perturbations, is 5 . Since one might be mainly interested in studying the perturbations of black holes in theories with the Gauss-Bonnet

\footnotetext{
${ }^{20}$ Of course, this logic reproduces the counting of operators in simpler EFTs based on a similar construction, such as [35].
} 
and/or the Chern-Simons operators, it is convenient to write the linearized action for the perturbations as

$$
\begin{aligned}
S=\int \mathrm{d}^{4} x \sqrt{-g}[ & \frac{M_{\mathrm{Pl}}^{2}}{2} R+\xi(r, \theta) \mathcal{G}+\zeta(r, \theta)^{*} R^{\mu \nu \rho \sigma} R_{\mu \nu \rho \sigma} \\
& \left.-\Lambda(r, \theta)+f_{1}(r, \theta) \delta g^{r r}+f_{2}(r, \theta) \delta g^{t t}+a f_{3}(r, \theta) \delta g^{t \phi}+a f_{4}(r, \theta) \delta g^{r \theta}\right],
\end{aligned}
$$

where $\xi$ and $\zeta$ are the coefficients of the Gauss-Bonnet and Chern-Simons operators. In general these can be arbitrary functions of $r$ and $\theta$, however in the subclass of shift-symmetric scalar theories (at the leading order in derivatives) each of them is proportional to the scalar field profile $\bar{\Phi}(r, \theta)$, hence they are identical up to a constant factor.

The Lagrangian (4.6) already captures many of the models that have been previously considered in the literature. However, at quadratic order in the perturbations, there can clearly be many other operators affecting the linearized dynamics. Finding a complete set is a cumbersome, but straightforward, procedure that closely follows our previous derivation in [35]. We will not derive it here in general, but, in the spirit of what we discussed in the previous sections, we want to emphasize that additional operators, such as $\left(\delta g^{r r}\right)^{2}$ or $\delta g^{r r} \delta K$, might not be completely irrelevant. In the presence of symmetries and with couplings satisfying the right power counting, they can be as large as the Gauss-Bonnet and the Chern-Simons operators on the considered background.

Note also that the generic angular dependence in equation (4.6), given the scalar profile (4.1), generically induces coupling terms between modes corresponding to different orbital numbers $\ell$, in an expansion in spherical harmonics. Differently from the mixing terms already discussed in section 3.2.2, the additional mixings induced by a non-trivial $\theta$ dependence couple modes with different $\ell$ within the odd (even) sector.

\section{Conclusions}

In this work we have introduced an effective field theory for perturbations of axisymmetric, slowly rotating spacetimes sourced by a scalar field coupled to gravity. Although our main focus was on fluctuations around asymptotically flat black holes with scalar hair, our construction is more general and applies, at the linear order in the spin parameter $a$, to any stationary, slowly rotating, axisymmetric background. For instance, it can be applied to study perturbations of the background describing the exterior of a star (once the boundary conditions at the surface of the object are specified) or even more exotic spacetimes supported by a scalar field [38].

To motivate the use of an effective approach for hairy black hole perturbations, we have discussed an explicit model where the Lagrangian contains, in addition to a coupling $\Phi \mathcal{G}$ between the scalar field $\Phi$ and the Gauss-Bonnet invariant $\mathcal{G}$, a higher derivative cubic galileon operator, $(\partial \Phi)^{2} \square \Phi$. Under the assumption that $(\partial \Phi)^{2} \square \Phi$ and $\Phi \mathcal{G}$ become strongly coupled at the same scale, we showed that the presence of $(\partial \Phi)^{2} \square \Phi$ in the theory yields order-one effects at the level of both the background solution and the dynamics of the perturbations, 
compared to the case where the coupling of this operator is set to zero. Many other operators, beyond our simple toy model, can induce similar effects at the level of the observables. In this sense, our effective theory is a convenient model-independent framework that allows to include all these effects in a single shot. We stress that, in our toy model, the introduction of the cubic galileon interaction is merely a choice. ${ }^{21}$ There are indications, however, that the presence of additional operators in the theory beyond just $\Phi \mathcal{G}$ is required by fundamental principles, such as microscopic causality and analyticity of the $S$-matrix [74].

There are a few interesting directions that we did not discuss here and we have left for future research. It would be interesting to use our effective theory to characterize more systematically the properties of the quasi-normal mode spectrum in theories beyond general relativity, and connect the deviations in the frequencies to the form of the operators in the EFT. We gave a simple example in this direction in section 2.2, where we showed that certain non-trivial relations among the effective couplings (see eqs. (2.35)), defining a specific subclass of theories within (2.28), allows to recover a degeneracy between the even and odd spectra, which is in general otherwise broken [10, 49]. Another possibility is along the lines of what we discussed in [35]. There, assuming small deviations from general relativity and introducing a light-ring expansion, we used the WKB formula of $[40,41]$ to infer some properties of the effective parameters from the spectrum. Our analysis applied, however, only to the case of a single Schrödinger-like equation. The result does not straightforwardly extend to a system of coupled equations, like the one we have in the even sector, or in theories with parity-breaking operators (see section 4 above). We will come back to this in a separate work [43]. ${ }^{22}$

Another important open question is about the generalization of the effective theory to rotating spacetimes, beyond the linear order in $a$. This would require revisiting our construction, identifying the new complete set of operators for the metric perturbations around the chosen ansatz for the rotating background metric (see, e.g., $[57,58])$ and understanding how to extend the Newman-Penrose formalism in the presence of an extra scalar field that couples to the graviton degrees of freedom to extract the linearized equations of motion.

It would also be interesting to apply our effective theory to more exotic spacetimes and check for instance how the conclusions of [38] (see also [75]) would get modified for a rotating background. ${ }^{23}$

\footnotetext{
${ }^{21}$ One could in principle set its coupling to a value that makes the operator negligible in the dynamics of the black hole, without running into fine tuning problems [45, 72, 73].

${ }^{22} \mathrm{~A}$ different way of characterizing deviations from general relativity in the QNM spectrum was proposed in $[28,29]$. In those works, the field potential in the Schrödinger-like equation is expanded as a power series in the radial coordinate, with small coefficients that embody generic deviations in both the background and the dynamics of the perturbations with respect to general relativity, without making any reference to an explicit Lagrangian.

${ }^{23}$ Note that, for symmetry reasons, at the linear order in $a$ the equation for the $\ell=0$ mode is unchanged with respect to the non-rotating case $(a=0)$, and therefore the conclusions of [38] would extend straightforwardly. This could change though for generic rotation, at nonlinear order in $a$.
} 


\section{Acknowledgments}

ET thanks Giovanni Maria Tomaselli for discussions. LH is supported by the DOE DESC0011941 and a Simons Fellowship in Theoretical Physics. AP is supported by the Simons Foundation Award No. 658906. LS is supported by the DOE DE-SC0011941 and the Simons Foundation Award No. 555117. ET is partly supported by the Italian MIUR under contract 2017FMJFMW (PRIN2017).

\section{A Black holes with galileon hair: odd sector}

The function $\mathcal{N}(r)$ appearing in eq. (2.26) is given by the solution of the following first-order differential equation,

$$
\begin{aligned}
& \mathcal{N}^{\prime}(r)=\frac{1}{4 r^{3}\left(r-r_{s}\right)\left(r-r_{s}+r \tilde{\alpha}^{2}(\mathcal{A}+\mathcal{B})\right)}\left[4 r^{2}\left(r-r_{s}\right)\left(r-2 r_{s}\right)\right. \\
& +\tilde{\alpha}^{2}\left[( r - r _ { s } ) \left(2 r _ { s } ^ { 2 } \left(3 g_{3} r^{2}\left(2 r^{2}-3 r r_{s}+r_{s}^{2}\right) \varphi^{\prime 3}+3 g_{3} r^{3}\left(r-r_{s}\right)^{2} \varphi^{\prime 2} \varphi^{\prime \prime}+4 r_{s}\left(11 r_{s}-8 r\right) \varphi^{\prime}\right.\right.\right. \\
& \left.\left.\left.\left.+8 r\left(r-r_{s}\right)\left(r_{s} \varphi^{\prime \prime}+r\left(r_{s}-r\right) \varphi^{\prime \prime \prime}\right)\right)+r^{4}\left(\mathcal{A}^{\prime}+\mathcal{B}^{\prime}\right)\right)+r^{3} \mathcal{A}\left(4 r-9 r_{s}\right)+r^{3}\left(10 r-9 r_{s}\right) \mathcal{B}\right]\right]
\end{aligned}
$$

where $\varphi, \mathcal{A}$ and $\mathcal{B}$ are defined in eqs. (2.14) and (2.13), and solve eqs. (2.15) and (2.17). The potential $V_{Q}(r)$ in eq. (2.24) is

$$
\begin{aligned}
& V_{Q}=\left(1-\frac{r_{s}}{r}\right)\left(\frac{\ell(\ell+1)}{r^{2}}-\frac{3 r_{s}}{r^{3}}\right)+\frac{\tilde{\alpha}^{2}}{r^{6}}\left[8 \omega^{2} r^{5} r_{s}^{2}\left(r-r_{s}\right) \varphi^{\prime \prime}+g_{3} r^{3} r_{s}^{2}\left(r-r_{s}\right)^{3} \varphi^{\prime 2} \varphi^{\prime \prime}\right. \\
& +g_{3} r^{2} r_{s}^{2}\left(r-r_{s}\right)\left(2 r^{2}-3 r r_{s}+r_{s}^{2}\right) \varphi^{\prime 3}+4 r_{s}^{2}\left(r-r_{s}\right)\left(2\left(\ell^{2}+\ell-2\right) r^{2}-3\left(\ell^{2}+\ell-3\right) r r_{s}-3 r_{s}^{2}\right) \varphi^{\prime} \\
& -8\left(\ell^{2}+\ell-2\right) r^{3} r_{s}^{2}\left(r-r_{s}\right) \varphi^{\prime \prime}+8\left(\ell^{2}+\ell-5\right) r^{2} r_{s}^{3}\left(r-r_{s}\right) \varphi^{\prime \prime}+r^{3} \mathcal{A}\left(\ell(\ell+1) r-3 r_{s}\right) \\
& \left.+2 r^{2} r_{s}^{2}\left(r-r_{s}\right)^{2}\left(\left(11 r_{s}-4 r\right) \varphi^{\prime \prime \prime}+2 r\left(r-r_{s}\right) \varphi^{(4)}\right)+3 r^{3}\left(r-r_{s}\right) \mathcal{B}(r)+30 r r_{s}^{4}\left(r-r_{s}\right) \varphi^{\prime \prime}\right] .
\end{aligned}
$$

Note that in the limit $\tilde{\alpha} \rightarrow 0$ one recovers the Regge-Wheeler potential [48] of massless odd spin- 2 modes in general relativity. In the eikonal limit, $\ell \rightarrow \infty$, the potential (A.2) considerably simplifies and reduces to

$$
\begin{aligned}
V_{Q}(\ell \rightarrow \infty)= & \left(1-\frac{r_{s}}{r}\right) \frac{\ell(\ell+1)}{r^{2}}+\frac{\tilde{\alpha}^{2}}{r^{5}}\left[8 \omega^{2} r^{4} r_{s}^{2}\left(r-r_{s}\right) \varphi^{\prime \prime}\right. \\
& \left.+\ell(\ell+1)\left(r^{3} \mathcal{A}+4 r_{s}^{2}\left(r-r_{s}\right)\left(2 r-3 r_{s}\right) \varphi^{\prime}-8 r r_{s}^{2}\left(r-r_{s}\right)^{2} \varphi^{\prime \prime}\right)\right]+\mathcal{O}\left(\ell^{0}\right),
\end{aligned}
$$

where we kept only terms up to the order $\mathcal{O}(\ell)$.

\section{B Radial foliation and geometric decomposition}

In this appendix, we recall the main ingredients that we used to construct the EFT in the main text. The notation mirrors the one of $[35,38]$. We assume here the $\Phi$ is a scalar, while we refer to section 4 for a discussion about the case in which parity is broken. 
The black hole metric breaks spatial translations, while retaining axial symmetry. Since the scalar $\Phi$ on the background depends only on $r$ at linear order in $a$, it is convenient to define a foliation of the spacetime with $\Phi=$ constant hypersurfaces. The unit vector orthogonal to the radial foliation is given by [35]

$$
n_{\mu}=\frac{\nabla_{\mu} \Phi}{\sqrt{\nabla_{\mu} \Phi \nabla^{\mu} \Phi}}
$$

satisfying $n_{\mu} n^{\mu}=1$. In analogy with the $\mathrm{ADM}$ decomposition in general relativity, one can define the induced metric

$$
h_{\mu \nu} \equiv g_{\mu \nu}-n_{\mu} n_{\nu} .
$$

In the unitary gauge, $n_{r}=N$ and $n_{a}=0$, and we can write the metric as:

$$
\mathrm{d} s^{2}=N^{2} \mathrm{~d} r^{2}+h_{a b}\left(\mathrm{~d} x^{a}+N_{a} \mathrm{~d} r\right)\left(\mathrm{d} x^{b}+N^{b} \mathrm{~d} r\right)
$$

where the Latin indices $a, b, c$ denote temporal and angular coordinates, while $N$ and $N^{a}$ are the lapse and the shift, respectively. Equivalently,

$$
g_{\mu \nu}=\left(\begin{array}{cc}
h_{a b} & N_{a} \\
N_{b} & N^{2}+N^{c} N_{c}
\end{array}\right), \quad h_{\mu \nu}=\left(\begin{array}{cc}
h_{a b} & N_{a} \\
N_{b} & N^{c} N_{c}
\end{array}\right) .
$$

By the definitions of $h_{\mu \nu}$ and $n_{\mu}$ the following orthogonality conditions follow:

$$
h_{\nu}^{\mu} n_{\mu}=0, \quad n^{\nu} \nabla_{\mu} n_{\nu}=0 .
$$

The extrinsic curvature is given by:

$$
K_{\mu \nu}=h_{\mu}^{\alpha} h_{\nu}^{\beta} \nabla_{\alpha} n_{\beta}=h_{\mu}^{\alpha} \nabla_{\alpha} n_{\nu}=\nabla_{\mu} n_{\nu}-n^{\alpha} n_{\mu} \nabla_{\alpha} n_{\nu}
$$

Two orthogonality conditions for $K_{\mu \nu}$ follow from (B.5) and the definition (B.6),

$$
n^{\mu} K_{\mu \nu}=n^{\nu} K_{\mu \nu}=0 .
$$

The temporal and angular components of the extrinsic curvature can be expressed as

$$
K_{a b}=\nabla_{a} n_{b}=-N \Gamma_{a b}^{r}=\frac{1}{2 N}\left(\partial_{r} h_{a b}-D_{a} N_{b}-D_{b} N_{a}\right),
$$

which is manifestly symmetric in $(a, b)$ and where $D_{a}$ is the covariant derivative acting on the $(2+1)$-dimensional hypersurface:

$$
D_{a} V_{b}=h_{a}^{\mu} h_{b}^{\nu} \nabla_{\mu} V_{\nu}
$$

From equation (B.6) it follows that the trace of the extrinsic curvature can be expressed as a total derivative:

$$
K=\nabla_{\mu} n^{\mu} .
$$




\section{B.1 Gauss-Codazzi equation}

The $(2+1)$-dimensional Riemann tensor,

$$
-\hat{R}_{\alpha \beta \mu \nu} V^{\alpha}=D_{\mu} D_{\nu} V_{\beta}-D_{\nu} D_{\mu} V_{\beta},
$$

can be related to the four-dimensional Riemann tensor and the extrinsic curvature through the Gauss-Codazzi equation

$$
h_{\mu}^{\tau} h_{\nu}^{\rho} h_{\beta}^{\sigma} R_{\alpha \sigma \tau \rho}=\hat{R}_{\alpha \beta \mu \nu}+K_{\mu \beta} K_{\nu \alpha}-K_{\nu \beta} K_{\mu \alpha},
$$

and the contracted form

$$
R=\hat{R}-K_{\mu \nu} K^{\mu \nu}+K^{2}-2 \nabla_{\mu}\left(K n^{\mu}-n^{\nu} \nabla_{\nu} n^{\mu}\right) .
$$

\section{B.2 Geometric quantities evaluated on the background}

On the background (3.5) we are considering there are no $(r, a)$ components and many simplifications occur. The shift are zero $\bar{N}_{a}=0$, while the lapse is $\bar{N}=1 / \sqrt{B}$.

The induced metric takes the simple form

$$
\bar{h}_{\mu \nu}=\left(\begin{array}{cc}
\bar{g}_{a b} & 0 \\
0 & 0
\end{array}\right),
$$

while the temporal and angular components of the extrinsic curvature are given by

$$
\bar{K}_{a b}=\frac{\sqrt{B}}{2} \partial_{r} \bar{h}_{a b}=\frac{\sqrt{B}}{2} \partial_{r} \bar{g}_{a b}
$$

We provide now some components of the Christoffel symbols evaluated on the background that will be useful in what follows. At zeroth order in $a$ we have:

$$
\begin{aligned}
\bar{\Gamma}_{t \phi}^{\alpha} & =\frac{1}{2} \bar{g}^{\alpha \sigma}\left(\partial_{t} \bar{g}_{\phi \sigma}+\partial_{\phi} \bar{g}_{t \sigma}-\partial_{\sigma} \bar{g}_{t \phi}\right)=0, \\
\bar{\Gamma}_{t r}^{\alpha} & =\frac{1}{2} \bar{g}^{\alpha \sigma}\left(\partial_{t} \bar{g}_{r \sigma}+\partial_{r} \bar{g}_{t \sigma}-\partial_{\sigma} \bar{g}_{t r}\right)=0, \quad \text { for } \alpha \neq t, \\
\bar{\Gamma}_{t r}^{t} & =\frac{1}{2} \bar{g}^{t t} \partial_{r} \bar{g}_{t t}, \\
\bar{\Gamma}_{\phi r}^{\alpha} & =\frac{1}{2} \bar{g}^{\alpha \sigma}\left(\partial_{\phi} \bar{g}_{r \sigma}+\partial_{r} \bar{g}_{\phi \sigma}-\partial_{\sigma} \bar{g}_{\phi r}\right)=0, \quad \text { for } \alpha \neq \phi, \\
\bar{\Gamma}_{\phi r}^{\phi} & =\frac{1}{2} \bar{g}^{\phi \phi} \partial_{r} \bar{g}_{\phi \phi},
\end{aligned}
$$

where we used that $\partial_{t} \bar{g}_{\mu \nu}=\partial_{\phi} \bar{g}_{\mu \nu}=0, \bar{g}_{r a}=0$ and $\bar{g}_{t \phi}=\mathcal{O}(a)$.

\section{Infinitesimal variations}

We collect here results on the infinitesimal variation of various tensors under an infinitesimal transformation $g_{\mu \nu} \rightarrow g_{\mu \nu}+\delta g_{\mu \nu}$. In this section all the terms without a $\delta$ in front are intended to be evaluated on the background. From $g_{\mu \alpha} g^{\alpha \nu}=\delta_{\mu}^{\nu}$ follows

$$
g^{\mu \nu} \delta g_{\mu \nu}=-g_{\mu \nu} \delta g^{\mu \nu}
$$


Moreover, from the relation for the determinant of an operator, $\log (\operatorname{det} M)=\operatorname{Tr}(\log M)$, it follows

$$
\delta g=g g^{\mu \nu} \delta g_{\mu \nu}=-g g_{\mu \nu} \delta g^{\mu \nu} .
$$

The variation of the volume element is easily computed as

$$
\delta \sqrt{-g}=\frac{1}{2} \sqrt{-g} g^{\mu \nu} \delta g_{\mu \nu}=-\frac{1}{2} \sqrt{-g} g_{\mu \nu} \delta g^{\mu \nu} .
$$

For the Christoffel symbol we obtain:

$$
\begin{aligned}
\delta \Gamma_{\mu \nu}^{\rho} & =\frac{1}{2} g^{\rho \sigma}\left(\nabla_{\mu} \delta g_{\nu \sigma}+\nabla_{\nu} \delta g_{\mu \sigma}-\nabla_{\sigma} \delta g_{\mu \nu}\right) \\
& =-\frac{1}{2} g^{\rho \sigma}\left(g_{\alpha \nu} g_{\beta \sigma} \nabla_{\mu}+g_{\alpha \mu} g_{\beta \sigma} \nabla_{\nu}-g_{\alpha \mu} g_{\beta \nu} \nabla_{\sigma}\right) \delta g^{\alpha \beta},
\end{aligned}
$$

which implies the variation of the Riemann tensor is

$$
\delta R_{\mu \rho \nu \sigma}=-R_{\beta \rho \nu \sigma} g_{\alpha \mu} \delta g^{\alpha \beta}+g_{\mu \lambda}\left(\nabla_{\nu} \delta \Gamma_{\rho \sigma}^{\lambda}-\nabla_{\sigma} \delta \Gamma_{\rho \nu}^{\lambda}\right)
$$

The variation of the Ricci tensor and its contracted version are:

$$
\begin{aligned}
\delta R_{\mu \nu} & =\nabla_{\rho}\left(\delta \Gamma_{\nu \mu}^{\rho}\right)-\nabla_{\nu}\left(\delta \Gamma_{\rho \mu}^{\rho}\right), \\
g^{\mu \nu} \delta R_{\mu \nu} & =\nabla^{\mu} \nabla^{\nu}\left(\delta g_{\mu \nu}\right)-\nabla^{\mu} g^{\alpha \beta} \nabla_{\mu}\left(\delta g_{\alpha \beta}\right)=\nabla^{\mu} v_{\mu}, \\
& =\left(-\nabla_{\mu} \nabla_{\nu}+g_{\mu \nu} \square\right) \delta g^{\mu \nu}
\end{aligned}
$$

which usually gives rise to boundary terms that can be neglected in a spacetime without boundaries.

For any scalar of the form

$$
X=\nabla_{\mu} X^{\mu}=\frac{1}{\sqrt{-g}} \partial_{\mu}\left(\sqrt{-g} X^{\mu}\right)
$$

the variation is

$$
\delta X=\frac{1}{2} g_{\mu \nu} \delta g^{\mu \nu} X+\nabla_{\mu}\left[\delta X^{\mu}-\frac{1}{2} X^{\mu} g_{\alpha \beta} \delta g^{\alpha \beta}\right] .
$$


For the foliation one has:

$$
\begin{aligned}
\delta n_{\mu}= & -\frac{1}{2} n_{\mu} n_{\alpha} n_{\beta} \delta g^{\alpha \beta}, \\
\delta n^{\mu}= & n_{\nu} \delta^{\mu \nu}-\frac{1}{2} n^{\mu} n_{\alpha} n_{\beta} \delta g^{\alpha \beta}, \\
\delta K_{\mu \nu}= & -\frac{1}{2} \nabla_{\mu}\left(n_{\nu} n_{\alpha} n_{\beta} \delta g^{\alpha \beta}\right) \\
& +\frac{1}{2}\left(g_{\alpha \nu} n_{\beta} \nabla_{\mu}+g_{\alpha \mu} n_{\beta} \nabla_{\nu}-g_{\alpha \mu} g_{\beta \nu} n^{\rho} \nabla_{\rho}\right) \delta g^{\alpha \beta} \\
& -n_{\beta} n_{\mu}\left(\nabla_{\alpha} n_{\nu}\right) \delta g^{\alpha \beta}+n_{\alpha} n_{\beta} n_{\mu} n^{\rho}\left(\nabla_{\rho} n_{\nu}\right) \delta g^{\alpha \beta} \\
& +\frac{1}{2} n_{\mu} n^{\rho} \nabla_{\rho}\left(n_{\nu} n_{\alpha} n_{\beta} \delta g^{\alpha \beta}\right)-\frac{1}{2} n_{\alpha} n_{\beta} n_{\mu} \nabla_{\nu} \delta g^{\alpha \beta}, \\
\delta K= & \frac{1}{2} g_{\mu \nu} \delta g^{\mu \nu} K+\nabla_{\mu}\left[n_{\nu} \delta g^{\mu \nu}-\frac{1}{2} n^{\mu}\left(n_{\alpha} n_{\beta}+g_{\alpha \beta}\right) \delta g^{\alpha \beta}\right], \\
\delta h^{\mu \nu}= & \delta g^{\mu \nu}-n_{\lambda}\left(n^{\mu} \delta g^{\nu \lambda}+n^{\nu} \delta g^{\mu \lambda}\right)+n^{\mu} n^{\nu} n_{\alpha} n_{\beta} \delta g^{\alpha \beta} \\
\delta \hat{R}= & \left(R_{\alpha \beta}-n^{\mu} n^{\nu} R_{\mu \alpha \nu \beta}-3 R_{\rho \alpha} n_{\beta} n^{\rho}+2 R_{\mu \nu} n^{\mu} n^{\nu} n_{\alpha} n_{\beta}\right) \delta g^{\alpha \beta} \\
& +h_{\mu}^{\nu} h^{\rho \sigma}\left(\nabla_{\nu} \delta \Gamma_{\rho \sigma}^{\mu}-\nabla_{\sigma} \delta \Gamma_{\rho \nu}^{\mu}\right)+2 K \delta K-2 K^{\mu \nu} \delta K_{\mu \nu} .
\end{aligned}
$$

The following identity is satisfied:

$$
n_{\mu} \delta h^{\mu \nu}=h^{\mu \nu} \delta n_{\mu}=0 .
$$

We provide an explicit expression for the variation of a component of the Christoffel symbol which will be of use in what follows:

$$
\begin{aligned}
\delta \Gamma_{t \phi}^{r} & =\frac{1}{2} g^{r \sigma}\left(\nabla_{t} \delta g_{\phi \sigma}+\nabla_{\phi} \delta g_{t \sigma}-\nabla_{\sigma} \delta g_{t \phi}\right) \\
& =\frac{1}{2} g^{r r}\left(\nabla_{t} \delta g_{\phi r}+\nabla_{\phi} \delta g_{t r}-\nabla_{t} \delta g_{t \phi}\right),
\end{aligned}
$$

where we used $\bar{g}_{r \sigma}=0$ for $\sigma \neq r$. At linear order in $a$, using the results of equation (B.16) we obtain:

$$
\begin{aligned}
\nabla_{t} \delta g_{\phi r} & =\partial_{t} \delta g_{\phi r}-\bar{\Gamma}_{t \phi}^{\alpha} \delta g_{\alpha r}-\bar{\Gamma}_{t r}^{\alpha} \delta g_{\phi \beta} \\
& =\partial_{t} \delta g_{\phi r}-\bar{\Gamma}_{t r}^{t} \delta g_{\phi t}, \\
\nabla_{\phi} \delta g_{t r} & =\partial_{\phi} \delta g_{t r}-\bar{\Gamma}_{\phi t}^{\alpha} \delta g_{\alpha r}-\bar{\Gamma}_{\phi r}^{\alpha} \delta g_{t \beta} \\
& =\partial_{\phi} \delta g_{t r}-\bar{\Gamma}_{\phi r}^{\phi} \delta g_{t \phi}, \\
\nabla_{r} \delta g_{t \phi} & =\partial_{r} \delta g_{t \phi}-\bar{\Gamma}_{t r}^{\alpha} \delta g_{\alpha \phi}-\bar{\Gamma}_{t \phi}^{\alpha} \delta g_{r \beta} \\
& =\partial_{r} \delta g_{t \phi}-\bar{\Gamma}_{t r}^{t} \delta g_{t \phi} .
\end{aligned}
$$

Another useful expression is the variation of the Gauss-Bonnet operator:

$$
\begin{aligned}
\int \mathrm{d}^{4} x \sqrt{-g} \xi(r) \delta \mathcal{G}=\int \mathrm{d}^{4} x \sqrt{-g}[ & 2 \xi\left(R R_{\alpha \beta}-R_{\alpha}{ }^{\rho \nu \sigma} R_{\beta \rho \nu \sigma}\right)+4 \nabla_{\rho} \nabla_{\sigma}\left(\xi R_{\alpha}{ }^{\rho}{ }^{\sigma}{ }^{\sigma}\right) \\
& +8 \nabla_{\lambda} \nabla_{\beta}\left(\xi R_{\alpha}{ }^{\lambda}\right)-2 \nabla_{\alpha} \nabla_{\beta}(\xi R)-4 \nabla_{\lambda} \nabla^{\lambda}\left(\xi R_{\alpha \beta}\right) \\
& \left.-4 g_{\alpha \beta} \nabla_{\mu} \nabla_{\nu}\left(\xi R^{\mu \nu}\right)+2 g_{\alpha \beta} \nabla_{\lambda} \nabla^{\lambda}(\xi R)\right] \delta g^{\alpha \beta},
\end{aligned}
$$

where $\xi(r)$ is a generic $r$-dependent function and $\mathcal{G}$ is the Gauss-Bonnet invariant defined in $(2.2)$. 


\section{C.1 Equivalent expressions for the $t \phi$ tadpole}

We show here that the tadpole $\delta K_{t \phi}$ can be rewritten as $\delta g^{t \phi}$. By construction, the $t \phi$ indices in $\delta K_{t \phi}$ are implicitly contracted with $\bar{g}_{t \phi}$ or its derivative, which are of order $a$. Therefore, working at linear order in $a$ we can neglect all the terms of order $a$ in the expression for $\delta K_{t \phi}$ as a polynomial in the unit vector, the background metric and their derivatives. By definition, the extrinsic curvature is

$$
K_{t \phi}=\nabla_{t} n_{\phi}=\partial_{t} n_{\phi}-\Gamma_{t \phi}^{\alpha} n_{\alpha}
$$

Considering its perturbation, at zeroth order in $a$ we have:

$$
\delta K_{t \phi}=\partial_{t} \delta n_{\phi}-\delta \Gamma_{t \phi}^{\alpha} \bar{n}_{\alpha}-\bar{\Gamma}_{t \phi}^{\alpha} \delta n_{\alpha}=-\delta \Gamma_{t \phi}^{r} \bar{n}_{r}
$$

where the first term was eliminated by an integration by parts, using that the background is time independent, the unit vector was set on the background and the last term was set to zero due to eq. (B.16). Using the results of equations (C.17) and (C.18) and getting rid of terms with $t$ and $\phi$ derivatives, by an integration by parts we obtain:

$$
\delta K_{t \phi}=f_{1}(r) \partial_{r} \delta g_{t \phi}+f_{2}(r) \delta g_{t \phi} .
$$

At zeroth order in $a$ one has $\delta g_{t \phi}=\bar{g}_{t \alpha} \bar{g}_{\phi \beta} \delta g^{\alpha \beta}=\bar{g}_{t t} \bar{g}_{\phi \phi} \delta g^{t \phi}$. Integrating by part the radial derivative we finally arrive at the desired result, i.e., up to boundary terms,

$$
\delta K_{t \phi}=f_{3}(r) \delta g^{t \phi} .
$$

\section{Explicit example}

In this appendix we discuss an example of slowly rotating hairy black hole solution in a theory with no higher derivative operators, which can be described at quadratic order by the effective action of section 3.2.2. The theory is that of a minimally coupled phantom scalar field with negative kinetic term [76], described by the action

$$
S=\int \mathrm{d}^{4} x \sqrt{-g}\left[\frac{R}{2}+\frac{1}{2} g^{\mu \nu} \partial_{\mu} \Phi \partial_{\nu} \Phi-\mathcal{V}(\Phi)\right],
$$

where we have set $M_{\mathrm{Pl}}=1$ and where the potential is given by the expression

$$
\mathcal{V}(\Phi)=\frac{3(q+2 \mathcal{M})}{|q|^{3}}\left[\left(3+\Phi^{2}\right) \cdot \sinh |\Phi|-3|\Phi| \cdot \cosh \Phi\right] .
$$

Even if not well motivated from an EFT point of view due to its ghost-like kinetic term and the specific form of the potential, this theory serves the purpose of providing a simple example of hairy black hole which can be treated analytically. The spherically symmetric hairy black hole solution depends on two parameters, the asymptotic mass $8 \pi \mathcal{M}$ and the scalar charge $q$, and was found in ref. [76] to be:

$$
\begin{aligned}
& \bar{\Phi}(r)=\frac{q}{r}, \\
& A(r)=B(r)=\frac{r^{2}(6 \mathcal{M}+3 q) \mathrm{e}^{-\frac{q}{r}}}{4 q^{3}}-\left[\frac{r^{2}(6 \mathcal{M}+3 q)}{4 q^{3}}-\frac{r(6 \mathcal{M}+3 q)}{2 q^{2}}+\frac{6 \mathcal{M}+q}{2 q}\right] \mathrm{e}^{\frac{q}{r}}, \\
& C(r)=r^{2} \mathrm{e}^{-\frac{q}{r}} .
\end{aligned}
$$


This solution can be straightforwardly generalized to a slowly rotating hairy black hole. Using the background equation (3.15) derived in section 3.2.1, we find that at first order in $a$ the functions $\bar{\Phi}(r), A(r), B(r), C(r)$ are unmodified, while

$$
D(r)=\frac{6 \mathcal{M}}{4 q^{3}}\left[\left(2 q^{2}-2 q r+r^{2}\right) \mathrm{e}^{\frac{q}{r}}-r^{2} \mathrm{e}^{-\frac{q}{r}}\right] .
$$

This solution reduces to a slowly rotating Kerr black hole in the limit $q \rightarrow 0$.

Another closed form example of slowly rotating hairy black hole has been recently presented in ref. [59], generalizing a solution first found in ref. [6] in a theory with a scalar field minimally coupled to gravity and with no higher derivate operators. The no-hair theorem of Bekenstein [77] is evaded thanks to a negative potential, $\mathcal{V}(\Phi)<0$, which in general corresponds to a violation of the weak energy condition. We refer the reader to ref. [59] for the explicit form of the solution.

As expected, the background metric and the scalar hair profile in both these examples respect the form of our ansatz (3.5). Even though they are not particularly physical, being based on theories with undesirable properties, such as a negative kinetic term or potential, they can be thought of as illustrative examples that show how the EFT works in the simplest possible case. Indeed, the dynamics of these theories can be captured in a unified way by our EFT, and in particular by its simplest form, corresponding to the leading order in the derivative expansion detailed in section 3.2.2.

\section{E Vector and tensor spherical harmonics, and some useful identities}

In problems with spherical symmetry it is convenient to express quantities in terms of spherical coordinates and expand the angular dependence in terms of spherical harmonics. In the case of slowly rotating backgrounds, however, the spherical symmetry is weakly broken by the spin parameter $a$. Spherical harmonics can still be a useful basis of functions to parametrize the angular dependence, but symmetry breaking effects can in general induce mixing between different harmonics. Because of this it is useful to work in terms of an orthonormal basis of functions, so that it is straightforward to project on the relevant basis components when needed.

We briefly review the construction of vector and tensor spherical harmonics, following [48], to fix our normalizations (see also appendix A of ref. [78]). Let us consider the 2 -sphere $S^{2}$, parametrized by the coordinates $\theta, \phi$, with $\theta \in[0, \pi]$ and $\phi \in[0,2 \pi)$. The Latin indices $i, j, \ldots$ will run on $\theta, \phi$. We will use the standard metric and Levi-Civita tensors on the sphere:

$$
\gamma_{i j}=\left(\begin{array}{cc}
1 & 0 \\
0 & \sin ^{2} \theta
\end{array}\right), \quad \varepsilon_{i j}=\sin \theta\left(\begin{array}{cc}
0 & +1 \\
-1 & 0
\end{array}\right) .
$$

We denote the scalar spherical harmonics as $Y^{(\ell, m)}(\theta, \phi)$, defined as

$$
Y^{(\ell, m)}(\theta, \phi)=(-1)^{m} \sqrt{\frac{2 \ell+1}{4 \pi}} \sqrt{\frac{(\ell-m) !}{(\ell+m) !}} P^{(\ell, m)}(\cos \theta) \mathrm{e}^{i m \phi},
$$


where the associated Legendre functions are given by Rodrigues' formula:

$$
P^{(\ell, m)}(x)=\frac{1}{2^{\ell} \ell !}\left(1-x^{2}\right)^{\frac{m}{2}} \frac{\mathrm{d}^{\ell+m}}{\mathrm{~d} x^{\ell+m}}\left(x^{2}-1\right)^{\ell}
$$

and satisfy the associated Legendre equation. The spherical harmonics so defined are orthonormal when integrated on the solid angle with $\mathrm{d} \Omega=\sin \theta \mathrm{d} \theta \mathrm{d} \phi$ :

$$
\int \mathrm{d} \Omega Y^{(\ell, m)}(\theta, \phi)^{*} Y^{\left(\ell^{\prime}, m^{\prime}\right)}(\theta, \phi)=\delta_{\ell \ell^{\prime}} \delta_{m m^{\prime}} .
$$

An orthonormal basis of vector and tensor spherical harmonics is given by:

$$
\begin{aligned}
{ }^{(+)} Y_{i}^{(\ell, m)} & =\frac{1}{\sqrt{\ell(\ell+1)}} \nabla_{i} Y^{(\ell, m)}, \\
{ }^{(-)} Y_{i}^{(\ell, m)} & =\frac{1}{\sqrt{\ell(\ell+1)}} \varepsilon_{i}^{j} \nabla_{j} Y^{(\ell, m)}, \\
{ }^{(\operatorname{tr})} Y_{i j}^{(\ell, m)} & =\frac{1}{\sqrt{2}} \gamma_{i j} Y^{(\ell, m)}, \\
{ }^{(+)} Y_{i j}^{(\ell, m)} & =\frac{\sqrt{2}}{\sqrt{\ell(\ell+1)\left(\ell^{2}+\ell-2\right)}}\left(\nabla_{i} \nabla_{j} Y^{(\ell, m)}-\frac{1}{2} \gamma_{i j} \nabla^{k} \nabla_{k} Y^{(\ell, m)}\right), \\
{ }^{(-)} Y_{i j}^{(\ell, m)} & =\frac{1}{2}\left(\varepsilon_{i}{ }^{(+)} Y_{k j}^{(\ell, m)}+\varepsilon_{j}^{k}{ }^{(+)} Y_{k i}^{(\ell, m)}\right),
\end{aligned}
$$

with scalar product corresponding to a trace on the discrete variables and an integral on the solid angle for the continuous ones.

\section{E.1 Recurrence relations and other useful identities}

The (associated) Legendre functions and the spherical harmonics satisfy a rich set of recurrence relations and identities, see for instance [79]. When dealing with problems with spherical symmetry it is usually sufficient to consider the simpler identities satisfied by Legendre polynomials, corresponding (up to factors) to spherical harmonics with $m=0$. In the case of a (slowly) rotating background, spherical symmetry is broken explicitly and we will need more general identities on spherical harmonics in order to explicitly express angular functions as combinations of (scalar, vector or tensor) spherical harmonics.

Two useful recurrence relations can be derived through straightforward manipulations of the relations on associated Legendre functions in [79]:

$$
\begin{aligned}
\cos \theta Y^{(\ell, m)} & =\zeta_{\ell, m} Y^{(\ell-1, m)}+\zeta_{\ell+1, m} Y^{(\ell+1, m)}, \\
\sin \theta \frac{\partial}{\partial \theta} Y^{(\ell, m)} & =-(\ell+1) \zeta_{\ell, m} Y^{(\ell-1, m)}+\ell \zeta_{\ell+1, m} Y^{(\ell+1, m)},
\end{aligned}
$$

where for notational convenience we introduced the coefficients

$$
\zeta_{\ell, m}=\sqrt{\frac{(\ell+m)(\ell-m)}{(2 \ell+1)(2 \ell-1)}} .
$$


Other useful identities, relating $Y^{(\ell, m)}$ and its $\theta$-derivative to the $\theta$-derivative of $Y^{(\ell \pm 1, m)}$, can be proved by manipulating the generating function of the associated Legendre functions:

$$
g_{m}(x, t)=\frac{(2 m) !\left(1-x^{2}\right)^{\frac{m}{2}}}{2^{m}(m !)\left(1-2 x t+t^{2}\right)^{m+\frac{1}{2}}}=\sum_{s=0}^{\infty} P^{(s+m, m)}(x) t^{s} .
$$

In particular, by taking the $x$-derivative of $g_{m}(x, t)$ and combining the resulting recurrence relation with a linear combination of equations (E.6) and (E.7) and the derivative of equation (E.6), the following identities are obtained:

$$
\begin{aligned}
\sin ^{2} \theta Y^{(\ell, m)}= & \frac{m^{2}}{\ell(\ell+1)} Y^{(\ell, m)}+\frac{1}{\ell} \zeta_{\ell, m} \sin \theta \frac{\partial}{\partial \theta} Y^{(\ell-1, m)} \\
& -\frac{1}{\ell+1} \zeta_{\ell+1, m} \sin \theta \frac{\partial}{\partial \theta} Y^{(\ell+1, m)}, \\
\frac{1}{2} \sin 2 \theta \frac{\partial}{\partial \theta} Y^{(\ell, m)}= & \frac{m^{2}}{\ell(\ell+1)} Y^{(\ell, m)}+\frac{\ell+1}{\ell} \zeta_{\ell, m} \sin \theta \frac{\partial}{\partial \theta} Y^{(\ell-1, m)} \\
& +\frac{\ell}{\ell+1} \zeta_{\ell+1, m} \sin \theta \frac{\partial}{\partial \theta} Y^{(\ell+1, m)} .
\end{aligned}
$$

The identities reduce to well-known relations on Legendre polynomials $P^{(\ell)}(x)$ for $m=0$.

\section{F Gauge choice}

We decompose a generic metric perturbation around the background (3.5) in terms of vector and tensor harmonics, following [48]. Using a notation where a Greek index $\mu$ runs on the Boyer-Lindquist coordinates $(t, r, \theta, \phi)$, and suppressing the indices $(\ell, m)$ and the explicit coordinate dependence, but keeping in mind that the scalar functions $\tilde{\mathrm{h}}, \tilde{H}, \tilde{\mathcal{H}}, \tilde{\mathcal{K}}$ and $\tilde{G}$ depend on $(t, r)$ :

$$
\begin{aligned}
\delta g_{\mu \nu}^{(\text {odd })} & =\left(\begin{array}{ccc}
0 & 0 & \tilde{\mathrm{h}}_{0}{ }^{(-)} Y_{i}^{(\ell, m)} \\
0 & 0 & \tilde{\mathrm{h}}_{1}{ }^{(-)} Y_{i}^{(\ell, m)} \\
(\mathrm{sym}) & (\mathrm{sym}) & \tilde{\mathrm{h}}_{2}{ }^{(-)} Y_{i j}^{(\ell, m)}
\end{array}\right) \\
\delta g_{\mu \nu}^{(\text {even })} & =\left(\begin{array}{ccc}
\tilde{H}_{0} Y^{(\ell, m)} & \tilde{H}_{1} Y^{(\ell, m)} & \tilde{\mathcal{H}}_{0}{ }^{(+)} Y_{i}^{(\ell, m)} \\
(\mathrm{sym}) & \tilde{H}_{2} Y^{(\ell, m)} & \tilde{\mathcal{H}}_{1}{ }^{(+)} Y_{i}^{(\ell, m)} \\
(\mathrm{sym}) & (\mathrm{sym}) & \tilde{\mathcal{K}}^{(\operatorname{tr})} Y_{i j}^{(\ell, m)}+\tilde{G}^{(+)} Y_{i j}^{(\ell, m)}
\end{array}\right) .
\end{aligned}
$$

Under an infinitesimal diffeomorphism transformation $x_{\mu} \rightarrow x_{\mu}+\xi_{\mu}$ the metric perturbation transforms as $\delta g_{\mu \nu} \rightarrow \delta g_{\mu \nu}-\nabla_{\mu} \xi_{\nu}-\nabla_{\nu} \xi_{\mu}$. The displacement vector can be expanded in harmonics as: ${ }^{24}$

$$
\xi_{\mu}^{(\text {even })}=\left(\alpha Y^{(\ell, m)}, \beta Y^{(\ell, m)}, \gamma^{(+)} Y_{i}^{(\ell, m)}\right), \quad \xi_{\mu}^{(\text {odd })}=\left(0,0, \delta^{(-)} Y_{i}^{(\ell, m)}\right),
$$

\footnotetext{
${ }^{24}$ The function $\gamma$ that we use here to parametrize a gauge transformation of the even type should not be confused with the metric (E.1) in the previous section.
} 
where the functions $\alpha, \beta, \gamma, \delta$ have indices $(\ell, m)$ and depend on $(t, r)$. Since the background breaks explicitly rotational invariance and parity, mixing between parity-even and parityodd terms and mixing between different harmonics with angular number $\ell$ will be generated. For notational convenience we reintroduce the index $\ell$ to account for the mixing, but suppress the index $m$. All the metric functions and gauge parameters are understood to be functions of $(t, r)$, with time derivatives denoted by a dot and radial derivatives denoted by a prime. At linear order in the spin parameter $a$, by using the identities of appendix $\mathrm{E}$, it is straightforward if somewhat lengthy to derive how the metric perturbations transform under an infinitesimal diffeomorphism. We obtain:

$$
\begin{aligned}
& \tilde{\mathrm{h}}_{0}^{(\ell)} \rightarrow \tilde{\mathrm{h}}_{0}^{(\ell)}-\dot{\delta}^{(\ell)}-2 i m a \frac{1}{\ell(\ell+1)} D \delta^{(\ell)} \\
& +a \frac{1}{\ell} \zeta_{\ell, m} \sqrt{\ell(\ell+1)} B D^{\prime} \beta^{(\ell-1)}-a \frac{1}{\ell+1} \zeta_{\ell+1, m} \sqrt{\ell(\ell+1)} B D^{\prime} \beta^{(\ell+1)} \\
& -a \frac{2(\ell-1)}{\ell} \zeta_{\ell, m} \sqrt{\frac{\ell+1}{\ell-1}} \frac{D}{C} \gamma^{(\ell-1)}-a \frac{2(\ell+1)}{\ell+} \zeta_{\ell+1, m} \sqrt{\frac{\ell}{\ell+2}} \frac{D}{C} \gamma^{(\ell+1)}, \\
& \tilde{\mathrm{h}}_{1}^{(\ell)} \rightarrow \tilde{\mathrm{h}}_{1}^{(\ell)}-\delta^{\prime(\ell)}+\frac{C^{\prime}}{C} \delta^{(\ell)}-a \frac{1}{\ell} \zeta_{\ell, m} \sqrt{\ell(\ell+1)} \frac{D C^{\prime}-C D^{\prime}}{A C} \alpha^{(\ell-1)} \\
& +a \frac{1}{\ell+1} \zeta_{\ell+1, m} \sqrt{\ell(\ell+1)} \frac{D C^{\prime}-C D^{\prime}}{A C} \alpha^{(\ell+1)}, \\
& \tilde{\mathrm{h}}_{2}^{(\ell)} \rightarrow \tilde{\mathrm{h}}_{2}^{(\ell)}-\sqrt{2} \sqrt{\ell^{2}+\ell-2} \delta^{(\ell)}, \\
& \tilde{H}_{0}^{(\ell)} \rightarrow \tilde{H}_{0}^{(\ell)}-2 \dot{\alpha}^{(\ell)}+A^{\prime} B \beta^{(\ell)}, \\
& \tilde{H}_{1}^{(\ell)} \rightarrow \tilde{H}_{1}^{(\ell)}+\frac{A^{\prime}}{A} \alpha^{(\ell)}-\alpha^{(\ell)}-\dot{\beta}^{(\ell)}-i m a \frac{1}{\ell(\ell+1)} \frac{A D^{\prime}-A^{\prime} D}{A C} \gamma^{(\ell)} \\
& +a \frac{(\ell-1)}{\sqrt{\ell(\ell-1)}} \zeta_{\ell, m}\left(A D^{\prime}-A^{\prime} D\right) \delta^{(\ell-1)} \\
& -a \frac{(\ell+2)}{\sqrt{(\ell+1)(\ell+2)}} \zeta_{\ell+1, m}\left(A D^{\prime}-A^{\prime} D\right) \delta^{(\ell+1)}, \\
& \tilde{H}_{2}^{(\ell)} \rightarrow \tilde{H}_{2}^{(\ell)}-\frac{B^{\prime}}{B} \beta^{(\ell)}-2 \beta^{(\ell)}, \\
& \tilde{\mathcal{H}}_{0}^{(\ell)} \rightarrow \tilde{\mathcal{H}}_{0}^{(\ell)}-\sqrt{\ell(\ell+1)} \alpha^{(\ell)}-\dot{\gamma}^{(\ell)}-i m a \frac{1}{\sqrt{\ell(\ell+1)}} B D^{\prime} \beta^{(\ell)}-2 i m a \frac{1}{\ell(\ell+1)} \frac{D}{C} \gamma^{(\ell)} \\
& +2 a \zeta_{\ell, m} \frac{\sqrt{(\ell-1)(\ell+1)}}{\ell} D \delta^{(\ell-1)} \\
& +2 a \zeta_{\ell+1, m} \frac{\sqrt{\ell(\ell+2)}}{\ell+1} D \delta^{(\ell+1)} \\
& \tilde{\mathcal{H}}_{1}^{(\ell)} \rightarrow \tilde{\mathcal{H}}_{1}^{(\ell)}-\sqrt{\ell(\ell+1)} \beta^{(\ell)}-\gamma^{(\ell)}+\frac{C^{\prime}}{C} \gamma^{(\ell)}+i m a \frac{1}{\sqrt{\ell(\ell+1)}} \frac{D C^{\prime}-C D^{\prime}}{A C} \alpha^{(\ell)}, \\
& \tilde{\mathcal{K}}^{(\ell)} \rightarrow \tilde{\mathcal{K}}^{(\ell)}+\sqrt{2} \sqrt{\ell(\ell+1)} \gamma^{(\ell)}-\sqrt{2} B C^{\prime} \beta^{(\ell)}, \\
& \tilde{G}^{(\ell)} \rightarrow \tilde{G}^{(\ell)}-\sqrt{2} \sqrt{\ell^{2}+\ell-2} \gamma^{(\ell)} \text {. }
\end{aligned}
$$

As expected on symmetry grounds, at linear order in $a$ perturbations of a given parity and fixed $\ell$ can receive contributions from opposite parity gauge transformations with $\ell \pm 1$ and 
from the $\phi$-derivative of same parity gauge transformations. Moreover, at linear order in $a$ and neglecting parity-breaking contributions for simplicity, the scalar field perturbation transforms as

$$
\delta \Phi^{(\ell)} \rightarrow \delta \Phi^{(\ell)}+\beta^{(\ell)} \bar{\Phi}^{\prime}
$$

where we have again expanded a scalar perturbation in spherical harmonics and suppressed the $m$ index.

The notation used in this appendix is related to the notation used in the rest of the article (chosen to agree with that of ref. [35]) as follows:

$$
\begin{array}{rlrl}
\mathrm{h}_{0} & =\sqrt{\ell(\ell+1)} \tilde{\mathrm{h}}_{0}, & \mathrm{~h}_{1}=\sqrt{\ell(\ell+1)} \tilde{\mathrm{h}}_{1}, & \mathrm{~h}_{2}=\frac{\sqrt{2}}{\sqrt{\ell(\ell+1)\left(\ell^{2}+\ell-2\right)}} \tilde{\mathrm{h}}_{2}, \\
H_{0} & =\tilde{H}_{0} / A, & H_{1}=\tilde{H}_{1}, & H_{2}=B \tilde{H}_{2}, \\
\mathcal{H}_{0} & =\sqrt{\ell(\ell+1)} \tilde{\mathcal{H}}_{0}, & \mathcal{H}_{1}=\sqrt{\ell(\ell+1)} \tilde{\mathcal{H}}_{1}, & \\
G & =\frac{\sqrt{2}}{C \sqrt{\ell(\ell+1)\left(\ell^{2}+\ell-2\right)}} \tilde{G}, \quad \mathcal{K}=-\frac{\sqrt{\ell(\ell+1)}}{C \sqrt{2\left(\ell^{2}+\ell-2\right)}} \tilde{G}+\frac{1}{C \sqrt{2}} \tilde{\mathcal{K}} .
\end{array}
$$

We choose to work in the Regge-Wheeler-unitary gauge, fixing

$$
\delta \Phi=\mathcal{H}_{0}=G=\mathrm{h}_{2}=0 .
$$

The conditions on $\delta \Phi, G, \mathrm{~h}_{2}$ determine in a unique way $\beta^{(\ell)}, \gamma^{(\ell)}, \delta^{(\ell)}$ respectively. The condition on $\mathcal{H}_{0}$ fixes $\alpha^{(\ell)}$ in terms of $\beta^{(\ell)}, \gamma^{(\ell)}, \delta^{(\ell \pm 1)}$. Since the gauge fixing is complete - i.e., it determines the gauge parameters in a unique way without ambiguity — it is consistent to fix the gauge at the level of the action without losing any constraint, see for instance ref. [80].

\section{G Linearized equations of motion with even-odd mixing}

In this appendix we derive the equations of motion for the theory (3.17), where we retained only the operators at the leading order in the derivative expansion. Similar manipulations hold in the presence of other quadratic operators in the EFT.

Modes with $\ell \geq 2$. Let us start assuming $\ell$ generic and $\ell \geq 2$. We start from the parametrization (2.20), where $\delta g_{\mu \nu}^{\text {odd }}$ and $\delta g_{\mu \nu}^{\text {even }}$ are given in (2.21) and (2.22). Then, we shall fix the gauge where $\mathcal{H}_{0}=G=\mathrm{h}_{2}=0$ and expand the action up to quadratic order in the fields. ${ }^{25}$ From the quadratic action, we can compute the equations of motion for the remaining fields $H_{0}, H_{1}, H_{2}, \mathcal{H}_{1}, \mathcal{K}, \mathrm{h}_{0}$ and $\mathrm{h}_{1}$. To integrate out more easily the constraint variables, it is convenient to redefine the metric perturbation $H_{2}$ as follows [35, 38, 81, 82],

$$
H_{2}=\psi+\frac{2 \ell(\ell+1)}{B(r) C^{\prime}(r)} \mathcal{H}_{1}+\frac{2 C(r)}{B(r) C^{\prime}(r)} \mathcal{K}^{\prime} .
$$

\footnotetext{
${ }^{25}$ As discussed in appendix $\mathrm{F}$, it is consistent to fix the gauge $\mathcal{H}_{0}=G=\mathrm{h}_{2}=0$ directly at the level of the action (3.17).
} 
This allows to get rid of $\mathcal{H}_{1}^{\prime}$ and $\mathcal{K}^{\prime \prime}$ from the $H_{0}$ 's equation of motion. The resulting set of coupled equations is:

$$
\begin{aligned}
& 0=Y_{\ell m}\left[\mathcal{H}_{1}\left(2 \ell(\ell+1)\left(C^{\prime}-\frac{C A^{\prime}}{A}\right)-\frac{4 \ell(\ell+1)^{2} C}{B C^{\prime}}+2 a m \omega\left(\frac{D C^{\prime}}{A}-\frac{C D^{\prime}}{A}\right)\right)\right. \\
& +\mathcal{K}^{\prime}\left(-\frac{2 C^{2} A^{\prime}}{A}-\frac{4 \ell(\ell+1) C^{2}}{B C^{\prime}}+2 C C^{\prime}\right)+\mathcal{K}\left(\frac{4 C}{B}-\frac{2 \ell(\ell+1) C}{B}\right) \\
& -2 B C C^{\prime} \psi^{\prime}+\psi\left(-\frac{B C A^{\prime} C^{\prime}}{A}-3 B^{\prime} C^{\prime} C-2 \ell(\ell+1) C-2 B C C^{\prime \prime}\right) \\
& \left.+2 i a m H_{1}\left(\frac{D C^{\prime}}{A}-\frac{C D^{\prime}}{A}\right)\right]+2 a \sin \theta\left(\partial_{\theta} Y_{\ell m}\right)\left[\mathrm{h}_{0}^{\prime}\left(\frac{D C^{\prime}}{A}-\frac{C D^{\prime}}{A}\right)\right. \\
& \left.+\mathrm{h}_{0}\left(\frac{C^{\prime} D^{\prime}}{A}-\frac{D C^{\prime 2}}{A C}\right)+i \omega \mathrm{h}_{1}\left(\frac{C D^{\prime}}{A}-\frac{D C^{\prime}}{A}\right)\right] \text {, } \\
& 0=Y_{\ell m}\left[i a m \mathcal{K}^{\prime} \frac{C D^{\prime}}{2 A C^{\prime}}+i \omega \mathcal{K}\left(\frac{C A^{\prime}}{2 A^{2}}-\frac{C^{\prime}}{2 A}\right)+\psi(r)\left(\frac{i a m B D C^{\prime}}{4 A C}+\frac{i a m B D^{\prime}}{4 A}+\frac{i \omega B C^{\prime}}{2 A}\right)\right. \\
& +\mathcal{H}_{1} \ell(\ell+1)\left(\operatorname{iam}\left(\frac{D^{\prime}}{2 A C^{\prime}}+\frac{D}{2 A C}\right)+\frac{i \omega}{2 A}\right)+H_{0}\left(\frac{i a m D^{\prime}}{4 A}-\frac{i a m D C^{\prime}}{4 A C}\right) \\
& \left.+H_{1} \frac{\ell(\ell+1)}{2 A}+a \mathrm{~h}_{1} \cos \theta \frac{\ell(\ell+1) D}{A C}\right]+a \mathrm{~h}_{1} \sin \theta\left(\partial_{\theta} Y_{\ell m}\right) \frac{\ell(\ell+1) D}{2 A C}, \\
& 0=Y_{\ell m}\left[\psi\left(\frac{B^{2} C A^{\prime} C^{\prime}}{A}+B C B^{\prime} C^{\prime}+2 B^{2} C C^{\prime \prime}+\frac{16 B^{3} M_{2}^{4} C^{2}}{M_{\mathrm{Pl}}^{2}}\right)\right. \\
& +\mathcal{H}_{1}\left(2 \ell(\ell+1)\left(C B^{\prime}-\frac{B\left(C^{\prime 2}-2 C C^{\prime \prime}\right)}{C^{\prime}}+\frac{16 B^{2} M_{2}^{4} C^{2}}{M_{\mathrm{Pl}}^{2} C^{\prime}}\right)+\frac{2 a m \omega B}{A}\left(D C^{\prime}+D^{\prime} C\right)\right) \\
& +2 H_{0}^{\prime} B C C^{\prime}-2 H_{0} \ell(\ell+1) C-H_{1}\left(4 i \omega \frac{B C C^{\prime}}{A}+\frac{2 i a m B}{A}\left(C D^{\prime}+C^{\prime} D\right)\right) \\
& +\mathcal{K}^{\prime}\left(2 C^{2} B^{\prime}+\frac{32 B^{2} M_{2}^{4} C^{3}}{M_{\mathrm{Pl}}^{2} C^{\prime}}+\frac{4 B C^{2} C^{\prime \prime}}{C^{\prime}}-2 B C C^{\prime}\right) \\
& \left.+\mathcal{K}\left(-\frac{4 \omega^{2} C^{2}}{A}+2\left(\ell^{2}+\ell-2\right) C-\frac{4 a m \omega C D}{A}\right)-8 a \mathrm{~h}_{0} \cos \theta \frac{\ell(\ell+1) D}{A}\right] \\
& +\frac{2 a B}{A} \sin \theta\left(\partial_{\theta} Y_{\ell m}\right)\left[-i \omega h_{1}\left(D C^{\prime}+C D^{\prime}\right)\right. \\
& \left.+h_{0}^{\prime}\left(D C^{\prime}+C D^{\prime}\right)+h_{0}\left(C^{\prime} D^{\prime}-\frac{2 D A^{\prime} C^{\prime}}{A}-\frac{D C^{2}}{C}-\frac{2 \ell(\ell+1) D}{B}\right)\right], \\
& 0=Y_{\ell m}\left[\psi\left(-\ell(\ell+1) \frac{B\left(C A^{\prime}+A C^{\prime}\right)}{4 A C}+a m \omega \frac{B\left(D C^{\prime}+C D^{\prime}\right)}{4 A C}\right)\right. \\
& +\mathcal{H}_{1} \ell(\ell+1)\left(\frac{\omega^{2}}{2 A}-\frac{\ell(\ell+1) C A^{\prime}+\left(\ell^{2}+\ell-2\right) A C^{\prime}}{2 A C C^{\prime}}+a m \omega \frac{D C^{\prime}+C D^{\prime}}{2 A C C^{\prime}}\right)
\end{aligned}
$$




$$
\begin{aligned}
& -\frac{1}{2} \ell(\ell+1) H_{0}^{\prime}+H_{0}\left(\ell(\ell+1)\left(\frac{C^{\prime}}{4 C}-\frac{A^{\prime}}{4 A}\right)+a m \omega \frac{D C^{\prime}-C D^{\prime}}{4 A C}\right)+i \omega H_{1} \frac{\ell(\ell+1)}{2 A} \\
& \times \frac{C}{2 A C^{\prime}} \mathcal{K}^{\prime}\left(a m \omega D^{\prime}-\ell(\ell+1) A^{\prime}\right)+\frac{a m \omega}{2 A^{2}} \mathcal{K}\left(D A^{\prime}-A D^{\prime}\right)+i a \omega \mathrm{h}_{1} \cos \theta \frac{\ell(\ell+1) D}{A C} \\
& \left.-2 a \mathrm{~h}_{0}^{\prime} \cos \theta \frac{\ell(\ell+1) D}{A C}+a \mathrm{~h}_{0} \cos \theta \ell(\ell+1) \frac{C\left(D A^{\prime}-2 A D^{\prime}\right)+3 A D C^{\prime}}{A^{2} C^{2}}\right] \\
& +\frac{a}{2 A C} \sin \theta\left(\partial_{\theta} Y_{\ell m}\right)\left[i \ell(\ell+1) \omega D \mathrm{~h}_{1}-2 \ell(\ell+1) D \mathrm{~h}_{0}^{\prime}\right. \\
& \left.+\mathrm{h}_{0}\left(\frac{\ell(\ell+1) D A^{\prime}}{A}+\frac{2\left(\ell^{2}+\ell+1\right) D C^{\prime}}{C}-\left(\ell^{2}+\ell+2\right) D^{\prime}\right)\right] \text {, } \\
& 0=Y_{\ell m}\left[\mathcal{K}^{\prime \prime} \frac{C^{2} A^{\prime}}{2 A C^{\prime}}-\mathcal{K} \frac{\omega^{2} C}{2 A B}\right. \\
& +\frac{C}{4} \mathcal{K}^{\prime}\left(\frac{C^{\prime 2}\left(3 B A^{\prime}+A B^{\prime}\right)+2 B C^{\prime \prime}\left(A C^{\prime}-C A^{\prime}\right)+2\left(\ell^{2}+\ell-4\right) A C^{\prime}}{A B C^{\prime 2}}-\frac{4 a m \omega D}{A B C^{\prime}}-\frac{4 \omega^{2} C}{A B C^{\prime}}\right) \\
& +\frac{1}{4} \psi\left(\frac{C A^{\prime} B^{\prime}+B A^{\prime} C^{\prime}+A\left(2 B^{\prime} C^{\prime}+2 B C^{\prime \prime}+\ell^{2}+\ell-4\right)}{A}-\frac{2 a m \omega D}{A}-\frac{2 \omega^{2} C}{A}\right) \\
& +\frac{1}{4} \psi^{\prime}\left(\frac{B C A^{\prime}}{A}+B C^{\prime}\right)-H_{1}^{\prime}\left(\frac{i a m D}{2 A}+\frac{i \omega C}{A}\right) \\
& -\frac{1}{4} H_{1}\left(i \omega \frac{2 C B^{\prime}+2 B C^{\prime}}{A B}-i a m \frac{B D A^{\prime}-A D B^{\prime}-2 A B D^{\prime}}{A^{2} B}\right) \\
& \times \frac{\ell(\ell+1)}{4} \mathcal{H}_{1}\left(-\frac{2 C A^{\prime} C^{\prime \prime}}{A C^{\prime 2}}+\frac{A^{\prime}}{A}-\frac{4 \omega^{2} C}{A B C^{\prime}}+\frac{B^{\prime} C^{\prime}+2\left(B C^{\prime \prime}+\ell^{2}+\ell-4\right)}{B C^{\prime}}\right. \\
& \left.+\frac{a m \omega D}{\ell(\ell+1)}\left(\frac{A^{\prime}}{A^{2}}+\frac{B^{\prime} C^{\prime}-4 \ell(\ell+1)}{A B C^{\prime}}\right)\right)+\frac{1}{4} \mathcal{H}_{1}^{\prime}\left(\frac{2 \ell(\ell+1) C A^{\prime}}{A C^{\prime}}+\frac{2 a m \omega D}{A}\right) \\
& \left.+\frac{1}{2} C H_{0}^{\prime \prime}+\frac{1}{4} C H_{0}^{\prime}\left(\frac{2 A^{\prime}}{A}+\frac{B^{\prime}}{B}+\frac{C^{\prime}}{C}\right)-H_{0} \frac{\ell(\ell+1)}{4 B}-a \mathrm{~h}_{0} \cos \theta \frac{\ell(\ell+1) D}{A B C}\right] \\
& +\frac{a D}{2 A} \sin \theta\left(\partial_{\theta} Y_{\ell m}\right)\left[\mathrm{h}_{0}^{\prime \prime}+\mathrm{h}_{0}^{\prime}\left(-\frac{3 A^{\prime}}{2 A}+\frac{B^{\prime}}{2 B}-\frac{C^{\prime}}{C}+\frac{2 D^{\prime}}{D}\right)\right. \\
& +\mathrm{h}_{0}\left(\frac{A^{\prime} C^{\prime}}{2 A C}-\frac{A^{\prime} D^{\prime}}{A D}+\frac{A^{\prime 2}}{A^{2}}-\frac{B^{\prime} C^{\prime}}{2 B C}+\frac{2}{B C}-\frac{C^{\prime} D^{\prime}}{C D}+\frac{C^{\prime 2}}{C^{2}}-\frac{C^{\prime \prime}}{C}\right) \\
& \left.-i \omega \mathrm{h}_{1}^{\prime}-i \omega \mathrm{h}_{1}\left(\frac{A^{\prime}}{2 A}+\frac{B^{\prime}}{2 B}\right)\right] \text {, }
\end{aligned}
$$

which are obtained from the variation with respect to $H_{0}, H_{1}, H_{2}, \mathcal{H}_{1}$ and $\mathcal{K}$ respectively, and

$$
\begin{aligned}
0= & Y_{\ell m}\left[-\mathrm{h}_{0}^{\prime \prime} \frac{\ell(\ell+1)}{2 A}+\mathrm{h}_{0}^{\prime} \ell(\ell+1) \frac{B A^{\prime}-A B^{\prime}}{4 A^{2} B}+\mathrm{h}_{1}^{\prime} \frac{i \ell(\ell+1)}{2 A}\left(\omega+\frac{a m D}{C}\right)\right. \\
& +\mathrm{h}_{0} \frac{\ell(\ell+1)\left(A\left(B^{\prime} C^{\prime}+2\left(B C^{\prime \prime}+\ell^{2}+\ell-2\right)\right)-B A^{\prime} C^{\prime}\right)}{4 A^{2} B C}
\end{aligned}
$$




$$
\begin{aligned}
& +\mathrm{h}_{1}\left(\frac{i a m\left(-\ell(\ell+1) B C D A^{\prime}+\ell(\ell+1) A C D B^{\prime}+2\left(\ell^{2}+\ell+2\right) A B C D^{\prime}-4 A B D C^{\prime}\right)}{4 A^{2} B C^{2}}\right. \\
& \left.+\frac{i \omega\left(-\ell(\ell+1) B C A^{\prime}+\ell(\ell+1) A C B^{\prime}+2 \ell(\ell+1) A B C^{\prime}\right)}{4 A^{2} B C}\right) \\
& +a \mathcal{K}^{\prime \prime} \cos \theta \frac{C D^{\prime}}{A C^{\prime}}+a \mathcal{K}^{\prime} \cos \theta\left(\frac{D A^{\prime}}{2 A^{2}}+\frac{D B^{\prime}}{2 A B}+\frac{2 \ell(\ell+1) D}{A B C^{\prime}}-\frac{C C^{\prime \prime} D^{\prime}}{A C^{\prime 2}}+\frac{D C^{\prime \prime}}{A C^{\prime}}+\frac{D^{\prime}}{A}\right) \\
& -a \mathcal{K} \cos \theta \frac{\left(\ell^{2}+\ell+2\right) D}{A B C}+\frac{a B}{2 A} \psi^{\prime} \cos \theta\left(\frac{D C^{\prime}}{C}+D^{\prime}\right)+\frac{a}{2 A} H_{0}^{\prime} \cos \theta\left(\frac{D C^{\prime}}{C}-D^{\prime}\right) \\
& +\frac{a D}{A C} \psi \cos \theta\left(\ell(\ell+1)+\frac{B A^{\prime} C^{\prime}}{2 A}+B^{\prime} C^{\prime}+\frac{C B^{\prime} D^{\prime}}{2 D}+B C^{\prime \prime}\right) \\
& +a \mathcal{H}_{1}^{\prime} \cos \theta \ell(\ell+1) \frac{C D^{\prime}-D C^{\prime}}{A C C^{\prime}}+a \mathcal{H}_{1} \cos \theta \frac{\ell(\ell+1)}{A^{2} B C^{2} C^{\prime 2}}\left(B C D A^{\prime} C^{\prime 2}\right. \\
& \left.\left.+A\left(C C^{\prime}\left(D\left(B C^{\prime \prime}+2 \ell(\ell+1)\right)-2 B C^{\prime} D^{\prime}\right)-B C^{2} C^{\prime \prime} D^{\prime}+B D C^{\prime 3}\right)\right)\right] \\
& +\frac{a D}{A} \sin \theta\left(\partial_{\theta} Y_{\ell m}\right)\left[\mathcal{K}^{\prime}\left(\frac{A^{\prime}}{4 A}+\frac{B^{\prime}}{4 B}+\frac{\ell(\ell+1)}{B C^{\prime}}-\frac{C C^{\prime \prime} D^{\prime}}{2 D C^{\prime 2}}+\frac{C^{\prime \prime}}{2 C^{\prime}}+\frac{D^{\prime}}{2 D}\right)\right. \\
& +\mathcal{K}^{\prime \prime} \frac{C D^{\prime}}{2 D C^{\prime}}-\mathcal{K} \frac{2}{B C}+\psi\left(\frac{B A^{\prime} C^{\prime}}{4 A C}+\frac{B^{\prime} C^{\prime}}{2 C}+\frac{B^{\prime} D^{\prime}}{4 D}+\frac{B C^{\prime \prime}}{2 C}+\frac{\ell(\ell+1)}{2 C}\right) \\
& +\frac{\ell(\ell+1)}{2 C^{2}} \mathcal{H}_{1}\left(\frac{C A^{\prime}}{A}+\frac{C\left(B C^{\prime \prime}+2 \ell(\ell+1)\right)}{B C^{\prime}}-\frac{\left(\ell^{2}+\ell+2\right) C D^{\prime}}{\ell(\ell+1) D}-\frac{C^{2} C^{\prime \prime} D^{\prime}}{D C^{\prime 2}}+\frac{2 C^{\prime}}{\ell(\ell+1)}\right) \\
& \left.+\frac{\ell(\ell+1)}{2} \mathcal{H}_{1}^{\prime}\left(\frac{D^{\prime}}{D C^{\prime}}-\frac{1}{C}\right)+H_{0}^{\prime}\left(\frac{C^{\prime}}{4 C}-\frac{D^{\prime}}{4 D}\right)\right], \\
& 0=Y_{\ell m}\left[\frac{1}{2} \mathrm{~h}_{1}\left(\frac{2 a \ell(\ell+1) m \omega D}{A C}+\frac{\ell(\ell+1) \omega^{2}}{A}-\frac{\ell(\ell+1)\left(\ell^{2}+\ell-2\right)}{C}\right)\right. \\
& +\frac{i}{2} \mathrm{~h}_{0}^{\prime} \ell(\ell+1)\left(\frac{m a D}{A C}+\frac{\omega}{A}\right)-\frac{i}{2} \mathrm{~h}_{0}\left(\frac{a\left(\ell^{2}+\ell-2\right) m D C^{\prime}}{A C^{2}}+\frac{\left(2 a m D^{\prime}+\ell(\ell+1) \omega C^{\prime}\right)}{A C}\right) \\
& -i \omega a \mathcal{K}^{\prime} \cos \theta \frac{C D^{\prime}}{A C^{\prime}}+\frac{i \omega}{A} a \mathcal{K} \cos \theta\left(D^{\prime}-\frac{D A^{\prime}}{A}\right)-\frac{B}{2 A} i \omega a \psi \cos \theta\left(\frac{D C^{\prime}}{C}+D^{\prime}\right) \\
& \left.-i \omega a \mathcal{H}_{1} \cos \theta \frac{\ell(\ell+1) D^{\prime}}{A C^{\prime}}-a H_{1} \cos \theta \frac{\ell(\ell+1) D}{A C}+\frac{i \omega}{2} a H_{0} \cos \theta\left(\frac{D^{\prime}}{A}-\frac{D C^{\prime}}{A C}\right)\right] \\
& +\frac{a D}{4 A C} \sin \theta\left(\partial_{\theta} Y_{\ell m}\right)\left[-2 i \omega \mathcal{K}^{\prime} \frac{C^{2} D^{\prime}}{D C^{\prime}}+2 i \omega \mathcal{K} C\left(\frac{D^{\prime}}{D}-\frac{A^{\prime}}{A}\right)-2 \ell(\ell+1) H_{1}\right. \\
& \left.-i \omega \psi B\left(C^{\prime}+\frac{C D^{\prime}}{D}\right)-2 i \omega \ell(\ell+1) \mathcal{H}_{1} \frac{C D^{\prime}}{D C^{\prime}}+i \omega H_{0}\left(\frac{C D^{\prime}}{D}-C^{\prime}\right)\right],
\end{aligned}
$$

which follow instead from the variation with respect to $h_{0}$ and $h_{1}$, respectively. It is clear from the previous equations that, as opposed to the non-rotating case [35, 38], the even and odd equations are now coupled. The dependence on the spherical harmonics can be removed by acting on each equation with $\int \mathrm{d} \Omega Y_{\ell^{\prime} m^{\prime}}^{*}(\theta, \phi)$ and using the identities (E.6) and (E.7). It becomes then clear that the even-odd mixing terms in the previous equations 
are between modes of different parity whose azimuthal quantum numbers differ by 1 . In other words, at linear order in $a$, the coupling is between an even $\ell$-mode and an odd $(\ell \pm 1)$-mode, and vice versa [67]. It is then easy to find the combinations of the previous equations that are algebraic in the fields $H_{0}, H_{1}, \mathcal{H}_{0}$ and $\mathrm{h}_{0}$. Solving for these variables and plugging the solutions into the remaining independent equations, one finds the final set of coupled equations for the three propagating fields $\psi, \mathcal{K}$ and $\mathrm{h}_{1}$. Dropping the even-odd mixing, the equation for $h_{1}$ decouples and reduces to (3.18) in the main text. Note also that the same manipulations have been performed to obtain the systems of equations $(2.27)$ and (2.34), with the huge simplification that, in the context of (2.27) and (2.34), $a=0$ and there is no mixing between even and odd fields.

Modes with $\ell=1$. When $\ell=1$, only the scalar mode propagates, although its equation of motion can still couple to the odd $\ell=2$ metric fluctuations. To derive the linearized equation, one can proceed as before with the simplification now that, when $\ell=1, \mathcal{K}$ is redundant in the parametrization (2.22) and can be set to zero. Similar field redefinitions (see eq. (G.1)) and manipulations to the ones above allow to integrate out $H_{0}, H_{1}$ and $\mathcal{H}_{0}$, and find the equation of motion for the dynamical field $\psi$ (see also refs. [35, 38]).

Mode with $\ell=\mathbf{0}$. The monopole $\ell=0$ can be derived in complete analogy. Note that, since it describes a spherically symmetric perturbation, its equation of motion will not contain any linear term proportional to the spin parameter $a$. The field equation for $\ell=0$ will be therefore identical to the one derived in the non-rotating case in refs. [35, 38].

Open Access. This article is distributed under the terms of the Creative Commons Attribution License (CC-BY 4.0), which permits any use, distribution and reproduction in any medium, provided the original author(s) and source are credited.

\section{References}

[1] V. Baibhav et al., Probing the nature of black holes: deep in the mHz gravitational-wave sky, Exper. Astron. 51 (2021) 1385 [arXiv:1908.11390] [INSPIRE].

[2] M. Giesler, M. Isi, M.A. Scheel and S. Teukolsky, Black hole ringdown: the importance of overtones, Phys. Rev. X 9 (2019) 041060 [arXiv: 1903.08284] [INSPIRE].

[3] V. Baibhav, E. Berti and V. Cardoso, LISA parameter estimation and source localization with higher harmonics of the ringdown, Phys. Rev. D 101 (2020) 084053 [arXiv:2001.10011] [INSPIRE].

[4] L. Hui and A. Nicolis, No-hair theorem for the Galileon, Phys. Rev. Lett. 110 (2013) 241104 [arXiv: 1202.1296] [INSPIRE].

[5] T.P. Sotiriou and S.-Y. Zhou, Black hole hair in generalized scalar-tensor gravity, Phys. Rev. Lett. 112 (2014) 251102 [arXiv:1312.3622] [INSPIRE].

[6] C.A.R. Herdeiro and E. Radu, Asymptotically flat black holes with scalar hair: a review, Int. J. Mod. Phys. D 24 (2015) 1542014 [arXiv:1504.08209] [InSPIRE].

[7] P. Creminelli, N. Loayza, F. Serra, E. Trincherini and L.G. Trombetta, Hairy black-holes in shift-symmetric theories, JHEP 08 (2020) 045 [arXiv:2004.02893] [INSPIRE]. 
[8] P. Kanti, N.E. Mavromatos, J. Rizos, K. Tamvakis and E. Winstanley, Dilatonic black holes in higher curvature string gravity, Phys. Rev. D 54 (1996) 5049 [hep-th/9511071] [InSPIRE].

[9] T.P. Sotiriou and S.-Y. Zhou, Black hole hair in generalized scalar-tensor gravity: an explicit example, Phys. Rev. D 90 (2014) 124063 [arXiv:1408.1698] [INSPIRE].

[10] J.L. Blázquez-Salcedo et al., Perturbed black holes in Einstein-dilaton-Gauss-Bonnet gravity: stability, ringdown, and gravitational-wave emission, Phys. Rev. D 94 (2016) 104024 [arXiv: 1609.01286] [INSPIRE].

[11] G. Antoniou, A. Bakopoulos and P. Kanti, Black-hole solutions with scalar hair in Einstein-scalar-Gauss-Bonnet theories, Phys. Rev. D 97 (2018) 084037 [arXiv:1711.07431] [INSPIRE].

[12] H. Witek, L. Gualtieri, P. Pani and T.P. Sotiriou, Black holes and binary mergers in scalar Gauss-Bonnet gravity: scalar field dynamics, Phys. Rev. D 99 (2019) 064035 [arXiv: 1810.05177] [INSPIRE].

[13] J.F.M. Delgado, C.A.R. Herdeiro and E. Radu, Spinning black holes in shift-symmetric Horndeski theory, JHEP 04 (2020) 180 [arXiv: 2002.05012] [INSPIRE].

[14] T.L. Smith, A.L. Erickcek, R.R. Caldwell and M. Kamionkowski, The effects of Chern-Simons gravity on bodies orbiting the earth, Phys. Rev. D 77 (2008) 024015 [arXiv: 0708.0001] [INSPIRE].

[15] D. Grumiller and N. Yunes, How do black holes spin in Chern-Simons modified gravity?, Phys. Rev. D 77 (2008) 044015 [arXiv: 0711.1868] [INSPIRE].

[16] N. Yunes and C.F. Sopuerta, Perturbations of Schwarzschild black holes in Chern-Simons modified gravity, Phys. Rev. D 77 (2008) 064007 [arXiv:0712.1028] [INSPIRE].

[17] N. Yunes and F. Pretorius, Dynamical Chern-Simons modified gravity. I. Spinning black holes in the slow-rotation approximation, Phys. Rev. D 79 (2009) 084043 [arXiv:0902.4669] [INSPIRE].

[18] K. Konno, T. Matsuyama and S. Tanda, Rotating black hole in extended Chern-Simons modified gravity, Prog. Theor. Phys. 122 (2009) 561 [arXiv:0902.4767] [INSPIRE].

[19] V. Cardoso and L. Gualtieri, Perturbations of Schwarzschild black holes in dynamical Chern-Simons modified gravity, Phys. Rev. D 80 (2009) 064008 [Erratum ibid. 81 (2010) 089903] [arXiv:0907.5008] [INSPIRE].

[20] C. Molina, P. Pani, V. Cardoso and L. Gualtieri, Gravitational signature of Schwarzschild black holes in dynamical Chern-Simons gravity, Phys. Rev. D 81 (2010) 124021 [arXiv: 1004 .4007] [INSPIRE].

[21] P. Pani, C.F.B. Macedo, L.C.B. Crispino and V. Cardoso, Slowly rotating black holes in alternative theories of gravity, Phys. Rev. D 84 (2011) 087501 [arXiv:1109.3996] [InSPIRE].

[22] K. Yagi, N. Yunes and T. Tanaka, Slowly rotating black holes in dynamical Chern-Simons gravity: deformation quadratic in the spin, Phys. Rev. D 86 (2012) 044037 [Erratum ibid. 89 (2014) 049902] [arXiv: 1206.6130] [INSPIRE].

[23] L.C. Stein and K. Yagi, Parametrizing and constraining scalar corrections to general relativity, Phys. Rev. D 89 (2014) 044026 [arXiv: 1310.6743] [INSPIRE].

[24] M. Kimura, Stability analysis of Schwarzschild black holes in dynamical Chern-Simons gravity, Phys. Rev. D 98 (2018) 024048 [arXiv: 1807.05029] [INSPIRE]. 
[25] P. Wagle, N. Yunes and H.O. Silva, Quasinormal modes of slowly-rotating black holes in dynamical Chern-Simons gravity, arXiv:2103.09913 [INSPIRE].

[26] M. Srivastava, Y. Chen and S. Shankaranarayanan, Analytical computation of quasinormal modes of slowly rotating black holes in dynamical Chern-Simons gravity, Phys. Rev. D 104 (2021) 064034 [arXiv:2106.06209] [INSPIRE].

[27] N. Loutrel, N. Yunes and F. Pretorius, Parametrized post-Einsteinian framework for gravitational wave bursts, Phys. Rev. D 90 (2014) 104010 [arXiv:1404.0092] [INSPIRE].

[28] V. Cardoso, M. Kimura, A. Maselli, E. Berti, C.F.B. Macedo and R. McManus, Parametrized black hole quasinormal ringdown: decoupled equations for nonrotating black holes, Phys. Rev. D 99 (2019) 104077 [arXiv: 1901.01265] [INSPIRE].

[29] R. McManus, E. Berti, C.F.B. Macedo, M. Kimura, A. Maselli and V. Cardoso, Parametrized black hole quasinormal ringdown. II. Coupled equations and quadratic corrections for nonrotating black holes, Phys. Rev. D 100 (2019) 044061 [arXiv:1906.05155] [INSPIRE].

[30] K. Glampedakis and H.O. Silva, Eikonal quasinormal modes of black holes beyond general relativity, Phys. Rev. D 100 (2019) 044040 [arXiv:1906.05455] [INSPIRE].

[31] H.O. Silva and K. Glampedakis, Eikonal quasinormal modes of black holes beyond general relativity. II. Generalized scalar-tensor perturbations, Phys. Rev. D 101 (2020) 044051 [arXiv: 1912.09286] [INSPIRE].

[32] A. Maselli, P. Pani, L. Gualtieri and E. Berti, Parametrized ringdown spin expansion coefficients: a data-analysis framework for black-hole spectroscopy with multiple events, Phys. Rev. D 101 (2020) 024043 [arXiv: 1910.12893] [INSPIRE].

[33] S. Endlich, V. Gorbenko, J. Huang and L. Senatore, An effective formalism for testing extensions to general relativity with gravitational waves, JHEP 09 (2017) 122 [arXiv: 1704.01590] [INSPIRE].

[34] O.J. Tattersall, P.G. Ferreira and M. Lagos, General theories of linear gravitational perturbations to a Schwarzschild black hole, Phys. Rev. D 97 (2018) 044021 [arXiv: 1711.01992] [INSPIRE].

[35] G. Franciolini, L. Hui, R. Penco, L. Santoni and E. Trincherini, Effective field theory of black hole quasinormal modes in scalar-tensor theories, JHEP 02 (2019) 127 [arXiv:1810.07706] [INSPIRE].

[36] P.A. Cano, K. Fransen and T. Hertog, Ringing of rotating black holes in higher-derivative gravity, Phys. Rev. D 102 (2020) 044047 [arXiv:2005.03671] [INSPIRE].

[37] P.A. Cano, K. Fransen, T. Hertog and S. Maenaut, Gravitational ringing of rotating black holes in higher-derivative gravity, arXiv:2110.11378 [INSPIRE].

[38] G. Franciolini, L. Hui, R. Penco, L. Santoni and E. Trincherini, Stable wormholes in scalar-tensor theories, JHEP 01 (2019) 221 [arXiv:1811.05481] [INSPIRE].

[39] C. Cheung, P. Creminelli, A.L. Fitzpatrick, J. Kaplan and L. Senatore, The effective field theory of inflation, JHEP 03 (2008) 014 [arXiv:0709.0293] [INSPIRE].

[40] B.F. Schutz and C.M. Will, Black hole normal modes: a semianalytic approach, Astrophys. J. Lett. 291 (1985) L33 [INSPIRE]. 
[41] S. Iyer and C.M. Will, Black hole normal modes: a WKB approach. 1. Foundations and application of a higher order WKB analysis of potential barrier scattering, Phys. Rev. D 35 (1987) 3621 [INSPIRE].

[42] E. Seidel and S. Iyer, Black hole normal modes: a WKB approach. 4. Kerr black holes, Phys. Rev. D 41 (1990) 374 [INSPIRE].

[43] L. Hui, A. Podo, L. Santoni and E. Trincherini, in progress.

[44] A. Nicolis, R. Rattazzi and E. Trincherini, The Galileon as a local modification of gravity, Phys. Rev. D 79 (2009) 064036 [arXiv:0811.2197] [INSPIRE].

[45] J. Noller, L. Santoni, E. Trincherini and L.G. Trombetta, Black hole ringdown as a probe for dark energy, Phys. Rev. D 101 (2020) 084049 [arXiv:1911.11671] [InSPIRE].

[46] S.E. Perkins, R. Nair, H.O. Silva and N. Yunes, Improved gravitational-wave constraints on higher-order curvature theories of gravity, Phys. Rev. D 104 (2021) 024060 [arXiv:2104.11189] [INSPIRE].

[47] R. Nair, S. Perkins, H.O. Silva and N. Yunes, Fundamental physics implications for higher-curvature theories from binary black hole signals in the LIGO-Virgo catalog GWTC-1, Phys. Rev. Lett. 123 (2019) 191101 [arXiv:1905.00870] [INSPIRE].

[48] T. Regge and J.A. Wheeler, Stability of a Schwarzschild singularity, Phys. Rev. 108 (1957) 1063 [INSPIRE].

[49] A. Bryant, H.O. Silva, K. Yagi and K. Glampedakis, Eikonal quasinormal modes of black holes beyond general relativity. III. Scalar Gauss-Bonnet gravity, Phys. Rev. D 104 (2021) 044051 [arXiv: 2106.09657] [INSPIRE].

[50] R.A. Rosen and L. Santoni, Black hole perturbations of massive and partially massless spin-2 fields in (anti) de Sitter spacetime, JHEP 03 (2021) 139 [arXiv: 2010.00595] [INSPIRE].

[51] S. Chandrasekhar, The mathematical theory of black holes, Clarendon, Oxford, U.K. (1985).

[52] E. Berti, V. Cardoso and A.O. Starinets, Quasinormal modes of black holes and black branes, Class. Quant. Grav. 26 (2009) 163001 [arXiv:0905. 2975] [INSPIRE].

[53] K. Glampedakis, A.D. Johnson and D. Kennefick, Darboux transformation in black hole perturbation theory, Phys. Rev. D 96 (2017) 024036 [arXiv:1702.06459] [INSPIRE].

[54] D. Pirtskhalava, L. Santoni, E. Trincherini and P. Uttayarat, Inflation from Minkowski space, JHEP 12 (2014) 151 [arXiv:1410.0882] [INSPIRE].

[55] A. Adams, N. Arkani-Hamed, S. Dubovsky, A. Nicolis and R. Rattazzi, Causality, analyticity and an IR obstruction to UV completion, JHEP 10 (2006) 014 [hep-th/0602178] [INSPIRE].

[56] S.A. Teukolsky, The Kerr metric, Class. Quant. Grav. 32 (2015) 124006 [arXiv:1410.2130] [INSPIRE].

[57] T. Johannsen, Regular black hole metric with three constants of motion, Phys. Rev. D 88 (2013) 044002 [arXiv:1501.02809] [INSPIRE].

[58] Z. Carson and K. Yagi, Asymptotically flat, parameterized black hole metric preserving Kerr symmetries, Phys. Rev. D 101 (2020) 084030 [arXiv:2002.01028] [INSPIRE].

[59] A. Bakopoulos and T. Nakas, Analytic and asymptotically flat hairy (ultra-compact) black-hole solutions and their stability analysis, arXiv:2107.05656 [INSPIRE]. 
[60] E. Babichev and C. Charmousis, Dressing a black hole with a time-dependent Galileon, JHEP 08 (2014) 106 [arXiv: 1312.3204] [INSPIRE].

[61] E. Babichev, C. Charmousis and A. Lehébel, Asymptotically flat black holes in Horndeski theory and beyond, JCAP 04 (2017) 027 [arXiv: 1702.01938] [INSPIRE].

[62] M. Minamitsuji and H. Motohashi, Stealth Schwarzschild solution in shift symmetry breaking theories, Phys. Rev. D 98 (2018) 084027 [arXiv: 1809.06611] [inSPIRE].

[63] J. Ben Achour, H. Liu and S. Mukohyama, Hairy black holes in DHOST theories: exploring disformal transformation as a solution-generating method, JCAP 02 (2020) 023 [arXiv: 1910.11017] [INSPIRE].

[64] S.A. Teukolsky, Perturbations of a rotating black hole. I. Fundamental equations for gravitational electromagnetic and neutrino field perturbations, Astrophys. J. 185 (1973) 635 [INSPIRE].

[65] W.H. Press and S.A. Teukolsky, Perturbations of a rotating black hole. II. Dynamical stability of the Kerr metric, Astrophys. J. 185 (1973) 649 [INSPIRE].

[66] P. Pani, Advanced methods in black-hole perturbation theory, Int. J. Mod. Phys. A 28 (2013) 1340018 [arXiv: 1305.6759] [INSPIRE].

[67] S. Chandrasekhar and V. Ferrari, On the non-radial oscillations of slowly rotating stars induced by the Lense-Thirring effect, Proc. Roy. Soc. Lond. A 433 (1991) 423.

[68] Y. Kojima, Coupled pulsations between polar and axial modes in a slowly rotating relativistic star, Prog. Theor. Phys. 90 (1993) 977.

[69] P. Pani, V. Cardoso, L. Gualtieri, E. Berti and A. Ishibashi, Perturbations of slowly rotating black holes: massive vector fields in the Kerr metric, Phys. Rev. D 86 (2012) 104017 [arXiv: 1209.0773] [INSPIRE].

[70] R. Jackiw and S.Y. Pi, Chern-Simons modification of general relativity, Phys. Rev. D 68 (2003) 104012 [gr-qc/0308071] [INSPIRE].

[71] S. Alexander and N. Yunes, Chern-Simons modified general relativity, Phys. Rept. 480 (2009) 1 [arXiv:0907.2562] [INSPIRE].

[72] D. Pirtskhalava, L. Santoni, E. Trincherini and F. Vernizzi, Weakly broken Galileon symmetry, JCAP 09 (2015) 007 [arXiv:1505.00007] [INSPIRE].

[73] L. Santoni, E. Trincherini and L.G. Trombetta, Behind Horndeski: structurally robust higher derivative EFTs, JHEP 08 (2018) 118 [arXiv:1806.10073] [INSPIRE].

[74] F. Serra, J. Serra, E. Trincherini and L.G. Trombetta, in progress.

[75] S. Mironov, V. Rubakov and V. Volkova, More about stable wormholes in beyond Horndeski theory, Class. Quant. Grav. 36 (2019) 135008 [arXiv:1812.07022] [INSPIRE].

[76] H. Dennhardt and O. Lechtenfeld, Scalar deformations of Schwarzschild holes and their stability, Int. J. Mod. Phys. A 13 (1998) 741 [gr-qc/9612062] [INSPIRE].

[77] J.D. Bekenstein, Novel "no-scalar-hair" theorem for black holes, Phys. Rev. D 51 (1995) R6608 [INSPIRE].

[78] L. Hui, A. Joyce, R. Penco, L. Santoni and A.R. Solomon, Static response and Love numbers of Schwarzschild black holes, JCAP 04 (2021) 052 [arXiv: 2010.00593] [INSPIRE]. 
[79] G.B. Arfken, H.J. Weber and F.E. Harris, Legendre functions, in Mathematical methods for physicists, chapter 15, Elsevier, The Netherlands (2013), pg. 715.

[80] H. Motohashi, T. Suyama and K. Takahashi, Fundamental theorem on gauge fixing at the action level, Phys. Rev. D 94 (2016) 124021 [arXiv:1608.00071] [InSPIRE].

[81] T. Kobayashi, H. Motohashi and T. Suyama, Black hole perturbation in the most general scalar-tensor theory with second-order field equations I: the odd-parity sector, Phys. Rev. D 85 (2012) 084025 [Erratum ibid. 96 (2017) 109903] [arXiv: 1202.4893] [INSPIRE].

[82] T. Kobayashi, H. Motohashi and T. Suyama, Black hole perturbation in the most general scalar-tensor theory with second-order field equations II: the even-parity sector, Phys. Rev. D 89 (2014) 084042 [arXiv: 1402.6740] [INSPIRE]. 